\title{
SOBOLEV'S THEOREM AND DUALITY FOR HERZ-MORREY SPACES OF VARIABLE EXPONENT
}

\author{
Yoshihiro Mizuta and Takao Ohno \\ Hiroshima Institute of Technology, Department of Mechanical Systems Engineering \\ 2-1-1 Miyake Saeki-ku Hiroshima 731-5193, Japan; y.mizuta.5x@it-hiroshima.ac.jp \\ Oita University, Faculty of Education and Welfare Science \\ Dannoharu Oita-city 870-1192, Japan; t-ohno@oita-u.ac.jp
}

\begin{abstract}
In this paper, we consider the Herz-Morrey space $\mathcal{H}_{\left\{x_{0}\right\}}^{p(\cdot), \omega}(G)$ of variable exponent consisting of all measurable functions $f$ on a bounded open set $G \subset \mathbf{R}^{n}$ satisfying

$$
\|f\|_{\mathcal{H}_{\left\{x_{0}\right\}}^{p(\cdot), \omega}(G)}=\left(\int_{0}^{2 d_{G}}\left(\omega\left(x_{0}, r\right)\|f\|_{L^{p(\cdot)}\left(B\left(x_{0}, r\right) \backslash B\left(x_{0}, r / 2\right)\right)}\right)^{q} d r / r\right)^{1 / q}<\infty,
$$

and set $\mathcal{H}^{p(\cdot), q, \omega}(G)=\bigcap_{x_{0} \in G} \mathcal{H}_{\left\{x_{0}\right\}}^{p(\cdot), q, \omega}(G)$.

Our first aim in this paper is to give the boundedness of the maximal and Riesz potential operators in $\mathcal{H}^{p(\cdot), q, \omega}(G)$ when $q=\infty$.

In connection with $\mathcal{H}_{\left\{x_{0}\right\}}^{p(\cdot), \omega}(G)$ and $\mathcal{H}^{p(\cdot), q, \omega}(G)$, let us consider the families $\underline{\mathcal{H}}_{\left\{x_{0}\right\}}^{p(\cdot), \omega}(G)$, $\underline{\mathcal{H}}^{p(\cdot), q, \omega}(G), \overline{\mathcal{H}}_{\left\{x_{0}\right\}}^{p \cdot(,)}(G)$ and $\tilde{\mathcal{H}}^{p(\cdot), q, \omega}(G)$. Following Fiorenza-Rakotoson [18], Di Fratta-Fiorenza [17] and Gogatishvili-Mustafayev [19], we next discuss the duality properties among these HerzMorrey spaces.
\end{abstract}

\section{Introduction}

Let $\mathbf{R}^{n}$ denote the $n$-dimensional Euclidean space. We denote by $B(x, r)$ the open ball centered at $x$ of radius $r$, and by $|E|$ the Lebesgue measure of a measurable set $E \subset \mathbf{R}^{n}$.

It is well known that the maximal operator is bounded in the Lebesgue space $L^{p}\left(\mathbf{R}^{n}\right)$ if $p>1$ (see [34]). In [12], the boundedness of the maximal operator is still valid by replacing the Lebesgue space by several Morrey spaces; the original one was introduced by Morrey [30] to estimate solutions of partial differential equations; for Morrey spaces, we also refer to Peetre [32] and Nakai [31].

One of important applications of the boundedness of the maximal operator is Sobolev's inequality; in the classical case,

$$
\left\|I_{\alpha} * f\right\|_{L^{p^{\sharp}}\left(\mathbf{R}^{n}\right)} \leq C\|f\|_{L^{p}\left(\mathbf{R}^{n}\right)}
$$

for $f \in L^{p}\left(\mathbf{R}^{n}\right), 0<\alpha<n$ and $1<p<n / \alpha$, where $I_{\alpha}$ is the Riesz kernel of order $\alpha$ and $1 / p^{\sharp}=1 / p-\alpha / n$ (see, e.g. [2, Theorem 3.1.4]). Sobolev's inequality for Morrey spaces was given by Adams [1] (also [12]). Further, Sobolev's inequality was also studied on generalized Morrey spaces (see [31]). This result was extended to local and global Morrey type spaces by Burenkov, Gogatishvili, Guliyev and Mustafayev

doi:10.5186/aasfm.2014.3913

2010 Mathematics Subject Classification: Primary 31B15, 46E35.

Key words: Herz-Morrey spaces of variable exponent, maximal functions, Riesz potentials, Sobolev's inequality, Trudinger's inequality, duality. 
[8] (see also [7, 9, 10]). The local Morrey type spaces are also called Herz spaces introduced by Herz [23]. In our paper, those Morrey type spaces are referred to as Herz-Morrey spaces.

In [13], Diening showed that the maximal operator is bounded on the variable exponent Lebesgue space $L^{p(\cdot)}\left(\mathbf{R}^{n}\right)$ if the variable exponent $p(\cdot)$, which is a constant outside a ball, satisfies the locally log-Hölder condition and inf $p(x)>1$ (see condition (P2) in Section 2). In the mean time, variable exponent Lebesgue spaces were used to discuss nonlinear partial differential equations with non-standard growth condition. These spaces have attracted more and more attention, in connection with the study of elasticity and fluid mechanics; see [16,33]. On the other hand, variable exponent Morrey or Herz versions were discussed in [4, 5, 24, 26, 29].

Let $G$ be a bounded open set in $\mathbf{R}^{n}$, whose diameter is denoted by $d_{G}$. Let $\omega(\cdot, \cdot): G \times(0, \infty) \rightarrow(0, \infty)$ be a uniformly almost monotone function on $G \times(0, \infty)$ satisfying the uniformly doubling condition. For $x_{0} \in G, 0<q \leq \infty$ and a variable exponent $p(\cdot)$, we consider the Herz-Morrey space $\mathcal{H}_{\left\{x_{0}\right\}}^{p(\cdot), \omega}(G)$ of variable exponent consisting of all measurable functions $f$ on $G$ satisfying

$$
\|f\|_{\mathcal{H}_{\left\{x_{0}\right\}}^{p(\cdot), \omega}(G)}=\left(\int_{0}^{2 d_{G}}\left(\omega\left(x_{0}, r\right)\|f\|_{L^{p(\cdot)}\left(B\left(x_{0}, r\right) \backslash B\left(x_{0}, r / 2\right)\right)}\right)^{q} d r / r\right)^{1 / q}<\infty ;
$$

when $q=\infty$,

$$
\|f\|_{\mathcal{H}_{\left\{x_{0}\right\}}^{p(\cdot), \infty, \omega}(G)}=\sup _{0<r<d_{G}} \omega\left(x_{0}, r\right)\|f\|_{L^{p(\cdot)\left(B\left(x_{0}, r\right) \backslash B\left(x_{0}, r / 2\right)\right)}}<\infty .
$$

Set

$$
\mathcal{H}^{p(\cdot), q, \omega}(G)=\bigcap_{x_{0} \in G} \mathcal{H}_{\left\{x_{0}\right\}}^{p(\cdot, q, \omega}(G)
$$

whose norm is defined by

$$
\|f\|_{\mathcal{H}^{p(\cdot), q, \omega}(G)}=\sup _{x_{0} \in G}\|f\|_{\mathcal{H}_{\left\{x_{0}\right\}}^{p(\cdot), \omega}(G)} .
$$

In connection with $\mathcal{H}_{\left\{x_{0}\right\}}^{p(\cdot), q, \omega}(G)$, let us consider the families $\underline{\mathcal{H}}_{\left\{x_{0}\right\}}^{p(\cdot), q, \omega}(G)$ and $\overline{\mathcal{H}}_{\left\{x_{0}\right\}}^{p(\cdot), q, \omega}(G)$ of all functions $f$ on $G$ satisfying

$$
\|f\|_{\underline{\mathcal{H}}_{\left\{x_{0}\right\}}^{p(\cdot,, \omega}(G)}=\left(\int_{0}^{2 d_{G}}\left(\omega\left(x_{0}, r\right)\|f\|_{L^{p(\cdot)}\left(B\left(x_{0}, r\right)\right)}\right)^{q} \frac{d r}{r}\right)^{1 / q}<\infty
$$

and

$$
\|f\|_{\overline{\mathcal{H}}_{\left\{x_{0}\right\}}^{p(\cdot, q, \omega}(G)}=\left(\int_{0}^{2 d_{G}}\left(\omega\left(x_{0}, r\right)\|f\|_{L^{p(\cdot)}\left(G \backslash B\left(x_{0}, r\right)\right)}\right)^{q} \frac{d r}{r}\right)^{1 / q}<\infty,
$$

respectively. In the paper by Fiorenza and Rakotoson [18], the Herz-Morrey space $\underline{\mathcal{H}}_{\left\{x_{0}\right\}}^{p(\cdot,, \omega}(G)$ is referred to as the generalized Lorentz space denoted by $G \Gamma(p, q, \omega)$.

Note here that

$$
\underline{\mathcal{H}}_{\left\{x_{0}\right\}}^{p(\cdot), \omega}(G) \cup \overline{\mathcal{H}}_{\left\{x_{0}\right\}}^{p(\cdot), \omega}(G) \subset \mathcal{H}_{\left\{x_{0}\right\}}^{p(\cdot), q, \omega}(G) .
$$

Similarly we consider the space

$$
\underline{\mathcal{H}}^{p(\cdot), q, \omega}(G)=\bigcap_{x_{0} \in G} \underline{\mathcal{H}}_{\left\{x_{0}\right\}}^{p(\cdot,, \omega}(G),
$$


whose norm is defined by

$$
\|f\|_{\underline{\mathcal{H}}^{p(\cdot), q, \omega}(G)}=\sup _{x_{0} \in G}\|f\|_{\underline{\mathcal{H}}_{\left\{x_{0}\right\}}^{p(\cdot), \omega}(G)} .
$$

Our first aim in this paper is to establish the boundedness of the maximal operator and the Riesz potential operator in $\mathcal{H}^{p(\cdot), \infty, \omega}(G)$; when $q<\infty$, we refer to [27]. In the borderline case, Trudinger's exponential integrability is discussed.

Next, following Di Fratta-Fiorenza [17] and Gogatishvili-Mustafayev [19], we study the duality properties among those Herz-Morrey spaces. In particular, we show the associate spaces of $\underline{\mathcal{H}}_{\left\{x_{0}\right\}}^{p(\cdot), \infty}(G)$ and $\overline{\mathcal{H}}_{\left\{x_{0}\right\}}^{p(\cdot), \omega}(G)$, which give another characterizations of Morrey spaces by Adams-Xiao [3] (see also [20]).

\section{Preliminaries}

Throughout this paper, let $C$ denote various constants independent of the variables in question. The symbol $g \sim h$ means that $C^{-1} h \leq g \leq C h$ for some constant $C>1$. Set $A(x, r)=B(x, r) \backslash B(x, r / 2)$.

Consider a function $p(\cdot)$ on $G$ such that

(P1) $1<p^{-}:=\inf _{x \in G} p(x) \leq \sup _{x \in G} p(x)=: p^{+}<\infty$, and

(P2) $p(\cdot)$ is log-Hölder continuous, namely

$$
|p(x)-p(y)| \leq \frac{c_{p}}{\log \left(2 d_{G} /|x-y|\right)} \quad \text { for } x, y \in G
$$

with a constant $c_{p} \geq 0 ; p(\cdot)$ is referred to as a variable exponent.

We also consider the family $\Omega(G)$ of all positive functions $\omega(\cdot, \cdot): G \times(0, \infty) \rightarrow$ $(0, \infty)$ satisfying the following conditions:

$(\omega 0) \omega(x, 0)=\lim _{r \rightarrow+0} \omega(x, r)=0$ for all $x \in G$ or $\omega(x, 0)=\infty$ for all $x \in G$;

$(\omega 1) \omega(x, \cdot)$ is uniformly almost monotone on $(0, \infty)$, that is, there exists a constant $Q_{1}>0$ such that $\omega(x, \cdot)$ is uniformly almost increasing on $(0, \infty)$, that is,

$$
\omega(x, r) \leq Q_{1} \omega(x, s) \text { for all } x \in G \text { and } 0<r<s
$$

or $\omega(x, \cdot)$ is uniformly almost decreasing on $(0, \infty)$, that is,

$$
\omega(x, s) \leq Q_{1} \omega(x, r) \text { for all } x \in G \text { and } 0<r<s ;
$$

$(\omega 2) \omega(x, \cdot)$ is uniformly doubling on $(0, \infty)$, that is, there exists a constant $Q_{2}>0$ such that

$$
Q_{2}^{-1} \omega(x, r) \leq \omega(x, 2 r) \leq Q_{2} \omega(x, r) \quad \text { for all } x \in G \text { and } r>0 ; \text { and }
$$

$(\omega 3)$ there exists a constant $Q_{3}>0$ such that

$$
Q_{3}^{-1} \leq \omega(x, 1) \leq Q_{3} \quad \text { for all } x \in G .
$$

Then one can find constants $a, b>0$ and $C>1$ such that

$$
C^{-1} r^{a} \leq \omega(x, r) \leq C r^{-b}
$$

for all $x \in G$ and $0<r \leq d_{G}$.

For later use, it is convenient to note the following result, which is proved by (P1), (P2) and (2.1). 
Lemma 2.1. There exists a constant $C>0$ such that

$$
\omega(x, r)^{p(x)} \leq C \omega(x, r)^{p(y)}
$$

whenever $|x-y|<r \leq d_{G}$.

For a locally integrable function $f$ on $G$, set

$$
\|f\|_{L^{p(\cdot)}(G)}=\inf \left\{\lambda>0: \int_{G}\left(\frac{|f(y)|}{\lambda}\right)^{p(y)} d y \leq 1\right\}
$$

in what follows, set $f=0$ outside $G$. We denote by $L^{p(\cdot)}(G)$ the family of locally integrable functions $f$ on $G$ satisfying $\|f\|_{L^{p(\cdot)}(G)}<\infty$.

Lemma 2.2. Let $0<q<\infty$. Then

(1) $\int_{0}^{2 d_{G}}\left(\omega(x, r)\|f\|_{L^{p(\cdot)}(A(x, r))}\right)^{q} d r / r \sim \sum_{j=1}^{\infty}\left(\omega\left(x, 2^{-j+1} d_{G}\right)\|f\|_{L^{p(\cdot)}\left(A\left(x, 2^{-j+1} d_{G}\right)\right)}\right)^{q}$;

(2) $\int_{0}^{2 d_{G}}\left(\omega(x, r)\|f\|_{L^{p(\cdot)}(B(x, r))}\right)^{q} d r / r \sim \sum_{j=1}^{\infty}\left(\omega\left(x, 2^{-j+1} d_{G}\right)\|f\|_{L^{p(\cdot)}\left(B\left(x, 2^{-j+1} d_{G}\right)\right)}\right)^{q}$; and

(3) $\int_{0}^{2 d_{G}}\left(\omega(x, r)\|f\|_{L^{p(\cdot)}(G \backslash B(x, r))}\right)^{q} d r / r \sim \sum_{j=1}^{\infty}\left(\omega\left(x, 2^{-j} d_{G}\right)\|f\|_{L^{p(\cdot)}\left(G \backslash B\left(x, 2^{-j} d_{G}\right)\right)}\right)^{q}$ for all $x \in G$ and measurable functions $f$ on $G$.

Proof. We only prove (1), since the remaining assertions can be proved similarly. Since $A(x, r) \supset B(x, 3 t / 2) \backslash B(x, t)$ when $3 t / 2<r<2 t \leq 2 d_{G}$, we have by $(\omega 1)$ and ( $\omega 2)$ that

$$
\int_{3 t / 2}^{2 t}\left(\omega(x, r)\|f\|_{L^{p(\cdot)}(A(x, r))}\right)^{q} d r / r \geq C\left(\omega(x, t)\|f\|_{L^{p(\cdot)}(B(x, 3 t / 2) \backslash B(x, t))}\right)^{q}
$$

and similarly, we have

$$
\int_{t}^{3 t / 2}\left(\omega(x, r)\|f\|_{L^{p(\cdot)}(A(x, r))}\right)^{q} d r / r \geq C\left(\omega(x, t)\|f\|_{L^{p(\cdot)}(B(x, t) \backslash B(x, 3 t / 4))}\right)^{q} .
$$

Thus

$$
\int_{t}^{2 t}\left(\omega(x, r)\|f\|_{L^{p(\cdot)}(A(x, r))}\right)^{q} d r / r \geq C\left(\omega(x, t)\|f\|_{L^{p(\cdot)}(B(x, 3 t / 2) \backslash B(x, 3 t / 4))}\right)^{q} .
$$

Therefore, letting $3 t / 2=2^{-j+1} d_{G}$ for a positive integer $j$, we see that

$$
\int_{2^{-j} d_{G}}^{2^{-j+2} d_{G}}\left(\omega(x, r)\|f\|_{L^{p(\cdot)}(A(x, r))}\right)^{q} d r / r \geq C\left(\omega\left(x, 2^{-j+1} d_{G}\right)\|f\|_{L^{p(\cdot)}\left(A\left(x, 2^{-j+1} d_{G}\right)\right)}\right)^{q},
$$

so that

$$
\begin{aligned}
\int_{0}^{2 d_{G}}\left(\omega(x, r)\|f\|_{L^{p(\cdot)}(A(x, r))}\right)^{q} d r / r & \geq \frac{1}{2} \sum_{j=1}^{\infty} \int_{2^{-j} d_{G}}^{2^{-j+2} d_{G}}\left(\omega(x, r)\|f\|_{L^{p(\cdot)}(A(x, r))}\right)^{q} d r / r \\
& \geq C \sum_{j=1}^{\infty}\left(\omega\left(x, 2^{-j+1} d_{G}\right)\|f\|_{L^{p(\cdot)}\left(A\left(x, 2^{-j+1} d_{G}\right)\right)}\right)^{q} .
\end{aligned}
$$

The converse inequality is easily obtained. 
Further, we obtain the next result.

Lemma 2.3. Suppose $0<q \leq \infty$. If $\|f\|_{h^{p(\cdot), q, \omega}(G)} \leq 1$, then there exists a constant $C>0$ such that $\|f\|_{h^{p(\cdot), \infty, \omega}(G)} \leq C$, for $h=\mathcal{H}_{\left\{x_{0}\right\}}, \underline{\mathcal{H}}_{\left\{x_{0}\right\}}, \overline{\mathcal{H}}_{\left\{x_{0}\right\}}, \mathcal{H}, \underline{\mathcal{H}}$.

By Lemma 2.1, we have the following result.

Lemma 2.4. There is a constant $C>0$ such that

$$
\int_{B\left(x_{0}, r\right)}|f(y)|^{p(y)} d y \leq C \omega\left(x_{0}, r\right)^{-p\left(x_{0}\right)}
$$

when $x_{0} \in G, 0<r<d_{G}$ and $\omega\left(x_{0}, r\right)\|f\|_{L^{p(\cdot)}\left(B\left(x_{0}, r\right)\right)} \leq 1$.

Lemma 2.5. There is a constant $C>0$ such that

$$
\frac{1}{\left|A\left(x_{0}, r\right)\right|} \int_{A\left(x_{0}, r\right)}|f(y)| d y \leq C r^{-n / p\left(x_{0}\right)} \omega\left(x_{0}, r\right)^{-1}
$$

when $x_{0} \in G, 0<r<d_{G}$ and $\omega\left(x_{0}, r\right)\|f\|_{L^{p(\cdot)}\left(A\left(x_{0}, r\right)\right)} \leq 1$.

Proof. Fix $x_{0} \in G$ and $0<r<d_{G}$. Let $f$ be a nonnegative measurable function on $G$ satisfying $\omega\left(x_{0}, r\right)\|f\|_{L^{p(\cdot)}\left(A\left(x_{0}, r\right)\right)} \leq 1$. Then we have by (P2) and Lemmas 2.1 and 2.4,

$$
\begin{aligned}
& \frac{1}{\left|A\left(x_{0}, r\right)\right|} \int_{A\left(x_{0}, r\right)} f(y) d y \\
& \leq r^{-n / p\left(x_{0}\right)} \omega\left(x_{0}, r\right)^{-1}+\frac{1}{\left|A\left(x_{0}, r\right)\right|} \int_{A\left(x_{0}, r\right)} f(y)\left(\frac{f(y)}{r^{-n / p\left(x_{0}\right)} \omega\left(x_{0}, r\right)^{-1}}\right)^{p(y)-1} d y \\
& \leq r^{-n / p\left(x_{0}\right)} \omega\left(x_{0}, r\right)^{-1}+C\left(r^{-n / p\left(x_{0}\right)} \omega\left(x_{0}, r\right)^{-1}\right)^{1-p\left(x_{0}\right)} \frac{1}{\left|A\left(x_{0}, r\right)\right|} \int_{A\left(x_{0}, r\right)} f(y)^{p(y)} d y \\
& \leq C r^{-n / p\left(x_{0}\right)} \omega\left(x_{0}, r\right)^{-1}
\end{aligned}
$$

as required.

\section{Boundedness of the maximal operator for $q=\infty$}

Let us consider the following conditions: let $\eta \in \Omega(G)$ and $x_{0} \in G$.

( $\omega 3.1)$ There exists a constant $Q>0$ such that

$$
\int_{0}^{r} t^{n-n / p\left(x_{0}\right)} \omega\left(x_{0}, t\right)^{-1} \frac{d t}{t} \leq Q r^{n-n / p\left(x_{0}\right)} \eta\left(x_{0}, r\right)^{-1}
$$

for all $0<r \leq d_{G}$; and

$(\omega 3.2)$ there exists a constant $Q>0$ such that

$$
\int_{r}^{2 d_{G}} t^{-n / p\left(x_{0}\right)} \omega\left(x_{0}, t\right)^{-1} \frac{d t}{t} \leq Q r^{-n / p\left(x_{0}\right)} \eta\left(x_{0}, r\right)^{-1}
$$

for all $0<r \leq d_{G}$.

By the doubling condition on $\omega$, one notes from $(\omega 3.1)$ or $(\omega 3.2)$ that

$$
\omega\left(x_{0}, r\right)^{-1} \leq C \eta\left(x_{0}, r\right)^{-1} .
$$


Lemma 3.1. If ( $\omega 3.1)$ and ( $\omega 3.2)$ hold for all $x_{0} \in G$ with the same constant $Q$, then there is a constant $C>0$ such that

$$
\int_{B(x, r)}|f(y)| d y \leq C r^{n-n / p(x)} \eta(x, r)^{-1}
$$

and

$$
\int_{G \backslash B(x, r)}|f(y)||x-y|^{-n} d y \leq C r^{-n / p(x)} \eta(x, r)^{-1}
$$

for all $x \in G, 0<r \leq d_{G}$ and $f$ with $\|f\|_{\mathcal{H}^{p(\cdot), \infty, \omega}(G)} \leq 1$.

Proof. Let $f$ be a nonnegative measurable function on $G$ satisfying $\|f\|_{\mathcal{H}^{p(\cdot), \infty, \omega}(G)} \leq$ 1. By Lemma 2.5 and ( $\omega 3.1)$, we have

$$
\begin{aligned}
\int_{B(x, r)} f(y) d y & =\sum_{j=1}^{\infty} \int_{A\left(x, 2^{-j+1} r\right)} f(y) d y \leq C \sum_{j=1}^{\infty}\left(2^{-j} r\right)^{n-n / p(x)} \omega\left(x, 2^{-j} r\right)^{-1} \\
& \leq C r^{n-n / p(x)} \eta(x, r)^{-1} .
\end{aligned}
$$

Similarly, we obtain by use of Lemma 2.5 and $(\omega 3.2)$

$$
\begin{aligned}
\int_{G \backslash B(x, r)}|f(y)||x-y|^{-n} d y & \leq C \sum_{j \geq 1,2^{j-1} r \leq d_{G}}\left(2^{j} r\right)^{-n} \int_{A\left(x, 2^{j} r\right)} f(y) d y \\
& \leq C \sum_{\substack{j \geq 1,2^{j-1} r \leq d_{G}\\
}}\left(2^{j} r\right)^{-n / p(x)} \omega\left(x, 2^{j} r\right)^{-1} \\
& \leq C r^{-n / p(x)} \eta(x, r)^{-1},
\end{aligned}
$$

as required.

For a locally integrable function $f$ on $G$, the Hardy-Littlewood maximal operator $\mathcal{M}$ is defined by

$$
\mathcal{M} f(x)=\sup _{r>0} \frac{1}{|B(x, r)|} \int_{B(x, r)}|f(y)| d y
$$

recall that $f=0$ outside $G$. Now we state the celebrated result by Diening [13].

Lemma 3.2. The maximal operator $\mathcal{M}$ is bounded in $L^{p(\cdot)}(G)$, that is, there exists a constant $C>0$ such that

$$
\|\mathcal{M} f\|_{L^{p(\cdot)}(G)} \leq C\|f\|_{L^{p(\cdot)}(G)} .
$$

Theorem 3.3. If ( $\omega 3.1)$ and ( $\omega 3.2)$ hold for all $x_{0} \in G$ with the same constant $Q$, then the maximal operator $\mathcal{M}$ is bounded from $\mathcal{H}^{p(\cdot), \infty, \omega}(G)$ to $\mathcal{H}^{p(\cdot), \infty, \eta}(G)$.

Guliyev, Hasanov and Samko [21, 22] proved that if ( $\omega 3.2)$ holds for all $x_{0} \in G$ with the same constant $Q$, then the maximal operator $\mathcal{M}$ is bounded from $\underline{\mathcal{H}}^{p(\cdot), \infty, \omega}(G)$ to $\underline{\mathcal{H}}^{p(\cdot), \infty, \eta}(G)$ and if $(\omega 3.1)$ holds for $x_{0} \in G$, then the maximal operator $\mathcal{M}$ is bounded from $\overline{\mathcal{H}}_{\left\{x_{0}\right\}}^{p(\cdot), \infty, \omega}(G)$ to $\overline{\mathcal{H}}_{\left\{x_{0}\right\}}^{p(\cdot), \infty, \eta}(G)$.

Proof of Theorem 3.3. Let $f$ be a nonnegative measurable function on $G$ such that $\|f\|_{\mathcal{H}^{p(\cdot), \infty, \omega}(G)} \leq 1$. For $x \in G$ and $0<r<d_{G}$, it suffices to show that

$$
\|\mathcal{M} f\|_{L^{p(\cdot)}(A(x, r))} \leq C \eta(x, r)^{-1} .
$$

For this purpose, set

$$
f=f \chi_{G \backslash B(x, 2 r)}+f \chi_{B(x, 2 r) \backslash B(x, r / 4)}+f \chi_{B(x, r / 4)}=f_{1}+f_{2}+f_{3},
$$


where $\chi_{E}$ denotes the characteristic function of $E$. We note from Lemma 3.2 that

$$
\begin{aligned}
\left\|\mathcal{M} f_{2}\right\|_{L^{p(\cdot)}(A(x, r))} \leq & C\left\|f_{2}\right\|_{L^{p(\cdot)}(G)} \leq C\left\|f_{2}\right\|_{L^{p(\cdot)}(B(x, 2 r) \backslash B(x, r / 4))} \\
\leq & C\left\{\left\|f_{2}\right\|_{L^{p(\cdot)}(B(x, 2 r) \backslash B(x, r))}+\left\|f_{2}\right\|_{L^{p(\cdot)}(B(x, r) \backslash B(x, r / 2))}\right. \\
& \left.+\left\|f_{2}\right\|_{L^{p(\cdot)}(B(x, r / 2) \backslash B(x, r / 4))}\right\} \\
\leq & C \omega(x, r)^{-1} \leq C \eta(x, r)^{-1} .
\end{aligned}
$$

For $z \in A(x, r)$, Lemma 3.1 gives

$$
\mathcal{M} f_{3}(z) \leq C r^{-n} \int_{B(x, r / 4)} f(y) d y \leq C r^{-n / p(x)} \eta(x, r)^{-1}
$$

so that

$$
\left\|\mathcal{M} f_{3}\right\|_{L^{p(\cdot)}(A(x, r))} \leq C r^{-n / p(x)} \eta(x, r)^{-1}\|1\|_{L^{p(\cdot)}(A(x, r))} \leq C \eta(x, r)^{-1} .
$$

Moreover, Lemma 3.1 again gives

$$
\mathcal{M} f_{1}(z) \leq C \int_{G \backslash B(x, 2 r)} f(y)|x-y|^{-n} d y \leq C r^{-n / p(x)} \eta(x, r)^{-1}
$$

and hence

$$
\left\|\mathcal{M} f_{1}\right\|_{L^{p(\cdot)}(A(x, r))} \leq C r^{-n / p(x)} \eta(x, r)^{-1}\|1\|_{L^{p(\cdot)}(A(x, r))} \leq C \eta(x, r)^{-1},
$$

as required.

Remark 3.4. If the conditions on $\omega$ hold at $x_{0} \in G$ only, then one can see that $\mathcal{M}$ is bounded from $\mathcal{H}_{\left\{x_{0}\right\}}^{p(\cdot), \infty, \omega}(G)$ to $\mathcal{H}_{\left\{x_{0}\right\}}^{p(\cdot), \infty, \eta}(G)$.

Corollary 3.5. For bounded functions $\nu(\cdot): G \rightarrow(-\infty, \infty)$ and $\beta(\cdot): G \rightarrow$ $(-\infty, \infty)$, set $\omega(x, r)=r^{\nu(x)}\left(\log \left(2 d_{G} / r\right)\right)^{\beta(x)}$. If $-n / p^{+}<\nu^{-} \leq \nu^{+}<n\left(1-1 / p^{-}\right)$, then the maximal operator $\mathcal{M}$ is bounded in $\mathcal{H}^{p(\cdot), \infty, \omega}(G)$.

Define

$$
\omega_{*}(x, r)=\left(\int_{0}^{r} \omega(x, t)^{-1} \frac{d t}{t}\right)^{-1}
$$

and

$$
\omega^{*}(x, r)=\left(\int_{r}^{2 d_{G}} \omega(x, t)^{-1} \frac{d t}{t}\right)^{-1}
$$

for $x \in G$ and $0<r \leq d_{G}$.

Theorem 3.6. (1) If $\omega_{*}\left(\cdot, d_{G}\right)$ is bounded in $G$, then $\mathcal{H}^{p(\cdot), \infty, \omega}(G) \subset \underline{\mathcal{H}}^{p(\cdot) \infty, \omega_{*}}(G)$.

(2) For each $x_{0} \in G, \mathcal{H}_{\left\{x_{0}\right\}}^{p(\cdot), \infty, \omega}(G) \subset \overline{\mathcal{H}}_{\left\{x_{0}\right\}}^{p(\cdot) \infty, \omega^{*}}(G)$.

Proof. Let $f$ be a measurable function on $G$ such that $\|f\|_{\mathcal{H}^{p(\cdot), \infty, \omega}(G)} \leq 1$. We show only (1), because (2) can be proved similarly.

For (1), we see that

$$
\|f\|_{L^{p(\cdot)}(B(x, r))} \leq \sum_{j=1}^{\infty}\|f\|_{L^{p(\cdot)}\left(A\left(x, 2^{-j+1} r\right)\right)} \leq \sum_{j=1}^{\infty} \omega\left(x, 2^{-j} r\right)^{-1} \leq C \omega_{*}(x, r)^{-1}
$$

for all $x \in G$ and $0<r \leq d_{G}$, as required. 
Remark 3.7. Let $\omega(x, r)=\left(\log \left(2 d_{G} / r\right)\right)^{\beta(x)+1}$ for a bounded function $\beta(\cdot): G \rightarrow$ $(-\infty, \infty)$.

(1) If $\operatorname{ess~inf}_{x \in G} \beta(x)>0$, then

$$
\omega_{*}(x, r) \sim\left(\log \frac{2 d_{G}}{r}\right)^{\beta(x)}
$$

for all $x \in G$ and $0<r<d_{G}$; and

(2) if $\beta\left(x_{0}\right)<0$ for $x_{0} \in G$, then

$$
\omega^{*}\left(x_{0}, r\right) \sim\left(\log \frac{2 d_{G}}{r}\right)^{\beta\left(x_{0}\right)}
$$

for all $0<r<d_{G}$.

Remark 3.8. Let $\omega(x, r)=r^{\nu(x)}$ for a bounded function $\nu(\cdot): G \rightarrow(-\infty, \infty)$.

(1) If $\operatorname{ess}_{\sup } \operatorname{seG}_{x \in G} \nu(x)<0$, then

$$
\omega_{*}(x, r) \sim \omega(x, r)
$$

for all $x \in G$ and $0<r<d_{G}$; and

(2) if $\nu\left(x_{0}\right)>0$ for $x_{0} \in G$, then

$$
\omega^{*}\left(x_{0}, r\right) \sim \omega\left(x_{0}, r\right)
$$

for all $0<r<d_{G}$.

Corollary 3.9. (1) Suppose ( $\omega 3.1)$ and ( $\omega 3.2)$ hold for all $x_{0} \in G$ with the same constant $Q$. If $\omega_{*}\left(\cdot, d_{G}\right)$ is bounded in $G$, then the maximal operator $\mathcal{M}$ is bounded from $\mathcal{H}^{p(\cdot), \infty, \omega}(G)$ to $\underline{\mathcal{H}}^{p(\cdot), \infty, \omega_{*}}(G)$.

(2) If ( $\omega 3.1)$ and ( $\omega 3.2)$ hold for $x_{0} \in \bar{G}$, then the maximal operator $\mathcal{M}$ is bounded from $\mathcal{H}_{\left\{x_{0}\right\}}^{p(\cdot), \infty, \omega}(G)$ to $\overline{\mathcal{H}}_{\left\{x_{0}\right\}}^{p(\cdot), \infty, \omega^{*}}(G)$.

Remark 3.10. Let us consider a singular integral operator $T$ associated with a standard kernel $k(x, y)$ in [15, Section 6.3] such that

$$
|k(x, y)| \leq K_{1}|x-y|^{-n}
$$

for all $x, y \in \mathbf{R}^{n}$ and

$$
\|T f\|_{L^{p(\cdot)}\left(\mathbf{R}^{n}\right)} \leq K_{2}\|f\|_{L^{p(\cdot)}\left(\mathbf{R}^{n}\right)}
$$

for all $f \in L^{p(\cdot)}\left(\mathbf{R}^{n}\right)$.

If $(\omega 3.1)$ and $(\omega 3.2)$ hold for all $x_{0} \in G$ with the same constant $Q$, then every singular integral operator $T$ is bounded from $\mathcal{H}^{p(\cdot), \infty, \omega}(G)$ to $\mathcal{H}^{p(\cdot), \infty, \eta}(G)$.

\section{Sobolev's inequality for $q=\infty$}

We consider the following condition: let $\eta \in \Omega(G)$ and $x_{0} \in G$.

$(\omega 4.1)$ For $0<\alpha<n$, there exists a constant $Q>0$ such that

$$
\int_{r}^{2 d_{G}} t^{\alpha-n / p(x)} \omega(x, t)^{-1} \frac{d t}{t} \leq Q r^{\alpha-n / p(x)} \eta(x, r)^{-1}
$$

for all $0<r<d_{G}$.

As in the proof of Lemma 3.1, we have the following result. 
Lemma 4.1. If ( $\omega 4.1)$ holds for all $x_{0} \in G$ with the same constant $Q$, then there is a constant $C>0$ such that

$$
\int_{G \backslash B(x, r)}|x-y|^{\alpha-n}|f(y)| d y \leq C r^{\alpha-n / p(x)} \eta(x, r)^{-1}
$$

for all $x \in G, 0<r<d_{G}$ and $f$ with $\|f\|_{\mathcal{H}^{p(\cdot), \infty, \omega}(G)} \leq 1$.

For $0<\alpha<n$, the Riesz potential $I_{\alpha} f$ is defined by

$$
I_{\alpha} f(x)=I_{\alpha} * f(x)=\int_{G}|x-y|^{\alpha-n} f(y) d y
$$

for measurable functions $f$ on $G$; and define

$$
\frac{1}{p^{\sharp}(x)}=\frac{1}{p(x)}-\frac{\alpha}{n} .
$$

Let us begin with Sobolev's inequality proved by Diening [14, Theorem 5.2]:

Lemma 4.2. If $0<\alpha<n / p^{+}$, then there exists a constant $C>0$ such that

$$
\left\|I_{\alpha} f\right\|_{L^{p^{\sharp}(\cdot)(G)}} \leq C\|f\|_{L^{p(\cdot)}(G)}
$$

for all $f \in L^{p(\cdot)}(G)$.

Our result is stated in the following:

Theorem 4.3. Let $0<\alpha<n / p^{+}$. If $(\omega 3.1)$ and $(\omega 4.1)$ hold for all $x_{0} \in G$ with the same constant $Q$, then there exists a constant $C>0$ such that

$$
\left\|I_{\alpha} f\right\|_{\mathcal{H}^{p^{\sharp}(\cdot), \infty, \eta}(G)} \leq C\|f\|_{\mathcal{H}^{p(\cdot), \infty, \omega}(G)}
$$

for all $f \in \mathcal{H}^{p(\cdot), \infty, \omega}(G)$.

In view of Guliyev, Hasanov and Samko [21, 22], if ( $\omega 4.1)$ holds for all $x_{0} \in G$ with the same constant $Q$, then there exists a constant $C>0$ such that

$$
\left\|I_{\alpha} f\right\|_{\underline{\mathcal{H}}^{\sharp(\cdot), \infty, \eta}(G)} \leq C\|f\|_{\underline{\mathcal{P}}^{p(\cdot), \infty, \omega}(G)}
$$

for all $f \in \underline{\mathcal{H}}^{p(\cdot), \infty, \omega}(G)$ and if $(\omega 3.1)$ holds for $x_{0} \in G$, then there exists a constant $C>0$ (which may depend on $x_{0}$ ) such that

$$
\left\|I_{\alpha} f\right\|_{\overline{\mathcal{H}}_{\left\{x_{0}\right\}}^{p^{\sharp}(\cdot, \infty, \eta}(G)} \leq C\|f\|_{\overline{\mathcal{H}}_{\left\{x_{0}\right\}}^{p(\cdot), \infty, \omega}(G)}
$$

for all $f \in \overline{\mathcal{H}}_{\left\{x_{0}\right\}}^{p(\cdot), \infty, \omega}(G)$.

Proof of Theorem 4.3. Let $f$ be a nonnegative measurable function on $G$ such that $\|f\|_{\mathcal{H}^{p(\cdot), \infty, \omega}(G)} \leq 1$. For $x \in G$ and $0<r<d_{G}$, we have only to show the inequality

$$
\left\|I_{\alpha} f\right\|_{L^{\left.p^{\sharp} \cdot\right)(A(x, r))}} \leq C \eta(x, r)^{-1} .
$$

Set

$$
f=f \chi_{G \backslash B(x, 2 r)}+f \chi_{B(x, 2 r) \backslash B(x, r / 4)}+f \chi_{B(x, r / 4)}=f_{1}+f_{2}+f_{3},
$$

as before. We note from Lemma 4.2 that

$$
\begin{aligned}
\left\|I_{\alpha} f_{2}\right\|_{L^{p^{\sharp}(\cdot)}(A(x, r))} & \leq C\left\|f_{2}\right\|_{L^{p(\cdot)}(G)} \leq C\left\|f_{2}\right\|_{L^{p(\cdot)}(B(x, 2 r) \backslash B(x, r / 4))} \\
& \leq C \omega(x, r)^{-1} \leq C \eta(x, r)^{-1} .
\end{aligned}
$$


If $z \in A(x, r)$, then Lemma 3.1 gives

$$
I_{\alpha} f_{3}(z) \leq C r^{\alpha-n} \int_{B(x, r / 4)} f(y) d y \leq C r^{\alpha-n / p(x)} \eta(x, r)^{-1},
$$

so that

$$
\left\|I_{\alpha} f_{3}\right\|_{L^{p^{\sharp}(\cdot)(A(x, r))}} \leq C r^{\alpha-n / p(x)} \eta(x, r)^{-1}\|1\|_{L^{p^{\sharp} \cdot(\cdot)}(A(x, r))} \leq C \eta(x, r)^{-1} .
$$

Moreover, Lemma 4.1 gives

$$
I_{\alpha} f_{1}(z) \leq \int_{G \backslash B(x, 2 r)}|x-y|^{\alpha-n} f(y) d y \leq C r^{\alpha-n / p(x)} \eta(x, r)^{-1},
$$

so that

$$
\left\|I_{\alpha} f_{1}\right\|_{L^{p^{\sharp}(\cdot)(A(x, r))}} \leq C r^{\alpha-n / p(x)} \eta(x, r)^{-1}\|1\|_{L^{p^{\sharp} \cdot()}(A(x, r))} \leq C \eta(x, r)^{-1},
$$

as required.

Corollary 4.4. Let $0<\alpha<n / p^{+}$and let $\nu, \beta$ and $\omega$ be as in Corollary 3.5. If $\alpha-n / p^{+}<\nu^{-} \leq \nu^{+}<n\left(1-1 / p^{-}\right)$, then there exists a constant $C>0$ such that

$$
\left\|I_{\alpha} f\right\|_{\mathcal{H}^{p^{\sharp}(\cdot), \infty, \omega}(G)} \leq C\|f\|_{\mathcal{H}^{p(\cdot), \infty, \omega}(G)}
$$

for all $f \in \mathcal{H}^{p(\cdot), \infty, \omega}(G)$.

Corollary 4.5. Assume that $0<\alpha<n / p^{+}$.

(1) Suppose ( $\omega 3.1)$ and $(\omega 4.1)$ hold for all $x_{0} \in G$ with the same constant $Q$. If $\omega_{*}\left(\cdot, d_{G}\right)$ is bounded in $G$, then the operator $I_{\alpha}$ is bounded from $\mathcal{H}^{p(\cdot), \infty, \omega}(G)$ to $\overline{\mathcal{H}}^{p^{\sharp}(\cdot), \infty, \omega_{*}}(G)$.

(2) If ( $\omega 3.1)$ and $(\omega 4.1)$ hold for $x_{0} \in G$, then the operator $I_{\alpha}$ is bounded from $\mathcal{H}_{\left\{x_{0}\right\}}^{p(\cdot), \infty, \omega}(G)$ to $\overline{\mathcal{H}}_{\left\{x_{0}\right\}}^{p^{\sharp}(\cdot), \infty, \omega^{*}}(G)$.

\section{Exponential integrability for $q=\infty$}

Set

$$
E_{1}(x, t)=\exp \left(t^{q(x)}\right)-1,
$$

where $1 / p(x)+1 / q(x)=1$. For a locally integrable function $f$ on $G$, set

$$
\|f\|_{L^{E_{1}(G)}}=\inf \left\{\lambda>0: \int_{G} E_{1}\left(x, \frac{|f(y)|}{\lambda}\right) d y \leq 1\right\} .
$$

We denote by $L^{E_{1}}(G)$ the class of locally integrable functions $f$ on $G$ satisfying $\|f\|_{L^{E_{1}(G)}}<\infty$.

In connection with $\mathcal{H}^{p(\cdot), q, \omega}(G)$, let us consider $\mathcal{H}^{E_{1}, q, \omega}(G)$ of all functions $f$ satisfying

$$
\|f\|_{\mathcal{H}^{E_{1}, q, \omega}(G)}=\sup _{x_{0} \in G}\left(\int_{0}^{2 d_{G}}\left(\omega\left(x_{0}, r\right)\|f\|_{L^{E_{1}\left(A\left(x_{0}, r\right)\right)}}\right)^{q} \frac{d r}{r}\right)^{1 / q}<\infty .
$$

Similarly, we define $\underline{\mathcal{H}}^{E_{1}, q, \omega}(G)$ and $\overline{\mathcal{H}}_{\left\{x_{0}\right\}}^{E_{1}, \omega}(G)$.

\section{Lemma 5.1.}

$$
\|1\|_{L^{E_{1}(B(x, r))}} \sim(\log (1+1 / r))^{-1 / q(x)}
$$

for all $x \in G$ and $0<r<d_{G}$. 
Lemma 5.2. [28, Theorem 4.1, Corollary 4.2] If $\alpha \geq n / p^{-}$, then there exists a constant $C>0$ such that

$$
\left\|I_{\alpha} f\right\|_{L^{E_{1}(G)}} \leq C\|f\|_{L^{p(\cdot)}(G)}
$$

for all $f \in L^{p(\cdot)}(G)$.

Our result is stated in the following:

Theorem 5.3. Let $\alpha \geq n / p^{-}$.

(1) If ( $\omega 3.1)$ and ( $\omega 4.1)$ hold for all $x_{0} \in G$ with the same constant $Q$, then there exists a constant $C>0$ such that

$$
\left\|I_{\alpha} f\right\|_{\mathcal{H}^{E_{1}, \infty, \eta}(G)} \leq C\|f\|_{\mathcal{H}^{p(\cdot), \infty, \omega}(G)}
$$

for all $f \in \mathcal{H}^{p(\cdot), \infty, \omega}(G)$.

(2) If ( $\omega 4.1)$ holds for all $x_{0} \in G$ with the same constant $Q$, then there exists a constant $C>0$ such that

$$
\left\|I_{\alpha} f\right\|_{\underline{\mathcal{H}}^{E_{1}, \infty, \eta}(G)} \leq C\|f\|_{\underline{\mathcal{H}}^{p(\cdot), \infty, \omega}(G)}
$$

for all $f \in \underline{\mathcal{H}}^{p(\cdot), \infty, \omega}(G)$.

(3) If ( $\omega 3.1$ ) holds for $x_{0} \in G$, then there exists a constant $C>0$ (which may depend on $\left.x_{0}\right)$ such that

$$
\left\|I_{\alpha} f\right\|_{\overline{\mathcal{H}}_{\left\{x_{0}\right\}}^{E_{1}, \infty, \eta}(G)} \leq C\|f\|_{\overline{\mathcal{H}}_{\left\{x_{0}\right\}}^{p(\cdot), \infty, \omega}(G)}
$$

for all $f \in \overline{\mathcal{H}}_{\left\{x_{0}\right\}}^{p(\cdot), \infty, \omega}(G)$.

Proof. We give only a proof of assertion (1). Let $f$ be a nonnegative measurable function on $G$ such that $\|f\|_{\mathcal{H}^{(\cdot), \infty, \omega}(G)} \leq 1$. We have only to show the inequality

$$
\left\|I_{\alpha} f\right\|_{L^{E_{1}(A(x, r))}} \leq C \eta(x, r)^{-1}
$$

for all $x \in G$ and $0<r<d_{G}$. Set

$$
f=f \chi_{G \backslash B(x, 2 r)}+f \chi_{B(x, 2 r) \backslash B(x, r / 4)}+f \chi_{B(x, r / 4)}=f_{1}+f_{2}+f_{3},
$$

as before. We note from Lemma 5.2 that

$$
\left\|I_{\alpha} f_{2}\right\|_{L^{E_{1}(A(x, r))}} \leq C\left\|f_{2}\right\|_{L^{p(\cdot)}(B(x, 2 r) \backslash B(x, r / 4))} \leq C \eta(x, r)^{-1} .
$$

If $z \in A(x, r)$, then Lemma 3.1 gives

$$
I_{\alpha} f_{3}(z) \leq C r^{\alpha-n} \int_{B(x, r / 4)} f(y) d y \leq C \eta(x, r)^{-1}
$$

since $\alpha \geq n / p^{-}$, so that

$$
\left\|I_{\alpha} f_{3}\right\|_{L^{E_{1}(A(x, r))}} \leq C \eta(x, r)^{-1}\|1\|_{L^{E_{1}(A(x, r))}} \leq C \eta(x, r)^{-1}
$$

by Lemma 5.1. Moreover, Lemma 4.1 gives

$$
I_{\alpha} f_{1}(z) \leq C \int_{G \backslash B(x, 2 r)}|x-y|^{\alpha-n} f(y) d y \leq C \eta(x, r)^{-1}
$$

since $\alpha \geq n / p^{-}$, so that

$$
\left\|I_{\alpha} f_{1}\right\|_{L^{E_{1}(A(x, r))}} \leq C \eta(x, r)^{-1}\|1\|_{L^{E_{1}(A(x, r))}} \leq C \eta(x, r)^{-1},
$$

as required. 
Corollary 5.4. Let $\alpha \geq n / p^{-}$and let $\nu, \beta$ and $\omega$ be as in Corollary 3.5.

(1) When $\alpha-n / p^{+}<\nu^{-} \leq \nu^{+}<n\left(1-1 / p^{-}\right)$, there exists a constant $C>0$ such that

$$
\left\|I_{\alpha} f\right\|_{\mathcal{H}^{E_{1}, \infty, \omega}(G)} \leq C\|f\|_{\mathcal{H}^{p(\cdot), \infty, \omega}(G)}
$$

for all $f \in \mathcal{H}^{p(\cdot), \infty, \omega}(G)$.

(2) When $\alpha-n / p^{+}<\nu^{-}$, there exists a constant $C>0$ such that

$$
\left\|I_{\alpha} f\right\|_{\underline{\mathcal{H}}^{E_{1}, \infty, \omega}(G)} \leq C\|f\|_{\underline{\mathcal{H}}^{p(\cdot), \infty, \omega}(G)}
$$

for all $f \in \underline{\mathcal{H}}^{p(\cdot), \infty, \omega}(G)$.

(3) When $\nu\left(x_{0}\right)<n\left(1-1 / p\left(x_{0}\right)\right)$ for $x_{0} \in G$, there exists a constant $C>0$ (which may depend on $x_{0}$ ) such that

$$
\left\|I_{\alpha} f\right\|_{\overline{\mathcal{H}}_{\left\{x_{0}\right\}}^{E_{1}, \infty, \omega}(G)} \leq C\|f\|_{\overline{\mathcal{H}}_{\left\{x_{0}\right\}}^{p(\cdot), \infty, \omega}(G)}
$$

for all $f \in \overline{\mathcal{H}}_{\left\{x_{0}\right\}}^{p(\cdot), \infty, \omega}(G)$.

\section{Associate spaces of $\overline{\mathcal{H}}_{\left\{x_{0}\right\}}^{p(\cdot), \infty, \omega}(G)$}

Recall that for $x_{0} \in G$ and measurable functions $f$ on $G$,

$$
\|f\|_{\overline{\mathcal{H}}_{\left\{x_{0}\right\}}^{p(\cdot), \infty, \omega}(G)}=\sup _{0<t<d_{G}} \omega\left(x_{0}, t\right)\|f\|_{L^{p(\cdot)}\left(G \backslash B\left(x_{0}, t\right)\right)}
$$

and

$$
\|f\|_{\underline{\mathcal{H}}_{\left\{x_{0}\right\}}^{p(\cdot), \omega}(G)}=\int_{0}^{2 d_{G}} \omega\left(x_{0}, t\right)\|f\|_{L^{p(\cdot)}\left(B\left(x_{0}, t\right)\right)} \frac{d t}{t} .
$$

Remark 6.1. Let $x_{0} \in G$. Note here that if $\omega\left(x_{0}, 0\right)=\infty$, then $\|f\|_{\overline{\mathcal{H}}_{\left\{x_{0}\right\}}^{p(\cdot), \infty, \omega}(G)}<$ $\infty$ if and only if $f=0$ a.e. Hence we may assume that $\omega\left(x_{0}, 0\right)=0$ and then $\omega\left(x_{0}, \cdot\right)$ is uniformly almost increasing on $(0, \infty)$ when $\|f\|_{\overline{\mathcal{H}}_{\left\{x_{0}\right\}}^{p(\cdot), \infty, \omega}(G)}<\infty$.

By the above remark, in this section, suppose

$$
\omega(x, 0)=0 \quad \text { for all } x \in G .
$$

For $x \in G$ and $0<t<d_{G}$, we set

$$
p^{+}(B(x, t))=\sup _{y \in B(x, t)} p(y),
$$

as before. We define $1 / q(x)=1-1 / p(x)$.

Following Di Fratta and Fiorenza [17], we have the following Hölder type inequality for log-type weights.

Theorem 6.2. For $x_{0} \in G$, suppose

(w6.1) there exist constants $b, Q>0$ such that

$$
\begin{aligned}
& \int_{0}^{t}\left(\log \frac{2 d_{G}}{r}\right)^{-b p\left(x_{0}\right)-1} \omega\left(x_{0}, r\right)^{-p^{+}\left(B\left(x_{0}, t\right)\right)} \frac{d r}{r} \leq Q\left(\log \frac{2 d_{G}}{t}\right)^{-b p\left(x_{0}\right)} \omega\left(x_{0}, t\right)^{-p\left(x_{0}\right)} \\
& \quad \text { for all } 0<t<d_{G} .
\end{aligned}
$$


Then there exists a constant $C>0$ such that

$$
\int_{G}|f(x) g(x)| d x \leq C\|f\|_{\underline{\mathcal{H}}_{\left\{x_{0}\right\}}^{q \cdot \cdot, 1, \eta}(G)}\|g\|_{\overline{\mathcal{H}}_{\left\{x_{0}\right\}}^{p(\cdot), \infty, \omega}(G)}
$$

for all measurable functions $f$ and $g$ on $G$, where

$$
\eta\left(x_{0}, r\right)=\left(\log \frac{2 d_{G}}{r}\right)^{-1} \omega\left(x_{0}, r\right)^{-1} .
$$

Proof. Let $x_{0} \in G$. Let $f$ and $g$ be nonnegative measurable functions on $G$ such that $\|f\|_{\underline{\mathcal{H}}_{\left\{x_{0}\right\}}^{q(\cdot), 1, \eta}(G)} \leq 1$ and $\|g\|_{\overline{\mathcal{H}}_{\left\{x_{0}\right\}}^{p(\cdot), \infty, \omega}(G)} \leq 1$. We have by Fubini's theorem and Hölder's inequality

$$
\begin{aligned}
& \int_{G} f(x) g(x) d x \\
& =\int_{G} f(x) g(x)\left(b\left(\log \frac{2 d_{G}}{\left|x-x_{0}\right|}\right)^{-b} \int_{\left|x-x_{0}\right|}^{2 d_{G}}\left(\log \frac{2 d_{G}}{t}\right)^{b-1} \frac{d t}{t}\right) d x \\
& =b \int_{0}^{2 d_{G}}\left(\int_{B\left(x_{0}, t\right)} f(x) g(x)\left(\log \frac{2 d_{G}}{\left|x-x_{0}\right|}\right)^{-b} d x\right)\left(\log \frac{2 d_{G}}{t}\right)^{b-1} \frac{d t}{t} \\
& \leq C \int_{0}^{2 d_{G}}\|f\|_{L^{q(\cdot)}\left(B\left(x_{0}, t\right)\right)}\left\|g\left(\log \frac{2 d_{G}}{\left|\cdot-x_{0}\right|}\right)^{-b}\right\|_{L^{p(\cdot)\left(B\left(x_{0}, t\right)\right)}}\left(\log \frac{2 d_{G}}{t}\right)^{b-1} \frac{d t}{t} .
\end{aligned}
$$

Here it suffices to show

$$
\begin{aligned}
\left\|g\left(\log \frac{2 d_{G}}{\left|\cdot-x_{0}\right|}\right)^{-b}\right\|_{L^{p(\cdot)\left(B\left(x_{0}, t\right)\right)}} & \leq C\left(\log \frac{2 d_{G}}{t}\right)^{-b} \omega\left(x_{0}, t\right)^{-1} \\
& =C\left(\log \frac{2 d_{G}}{t}\right)^{-b+1} \eta\left(x_{0}, t\right)
\end{aligned}
$$

for $0<t<d_{G}$. In fact, we obtain

$$
\begin{aligned}
& \int_{B\left(x_{0}, t\right)}\left(\frac{g(x)}{\left(\log \left(2 d_{G} / t\right)\right)^{-b} \omega\left(x_{0}, t\right)^{-1}}\right)^{p(x)}\left(\log \frac{2 d_{G}}{\left|x-x_{0}\right|}\right)^{-b p(x)} d x \\
& \leq C \int_{B\left(x_{0}, t\right)}\left(\frac{g(x)}{\left(\log \left(2 d_{G} / t\right)\right)^{-b} \omega\left(x_{0}, t\right)^{-1}}\right)^{p(x)}\left(\log \frac{2 d_{G}}{\left|x-x_{0}\right|}\right)^{-b p\left(x_{0}\right)} d x \\
& \leq C \int_{B\left(x_{0}, t\right)}\left(\frac{g(x)}{\left(\log \left(2 d_{G} / t\right)\right)^{-b} \omega\left(x_{0}, t\right)^{-1}}\right)^{p(x)}\left(\int_{0}^{\left|x-x_{0}\right|}\left(\log \frac{2 d_{G}}{r}\right)^{-b p\left(x_{0}\right)-1} \frac{d r}{r}\right) d x \\
& \leq C \int_{0}^{t}\left(\int_{B\left(x_{0}, t\right) \backslash B\left(x_{0}, r\right)} g(x)^{p(x)}\left(\log \frac{2 d_{G}}{t}\right)^{b p(x)} \omega\left(x_{0}, t\right)^{p(x)}\left(\log \frac{2 d_{G}}{r}\right)^{-b p\left(x_{0}\right)-1} d x\right) \frac{d r}{r}
\end{aligned}
$$




$$
\begin{aligned}
\leq & C\left(\log \frac{2 d_{G}}{t}\right)^{b p\left(x_{0}\right)} \omega\left(x_{0}, t\right)^{p\left(x_{0}\right)} \int_{0}^{t}\left(\log \frac{2 d_{G}}{r}\right)^{-b p\left(x_{0}\right)-1} \\
& \cdot\left(\int_{B\left(x_{0}, t\right) \backslash B\left(x_{0}, r\right)}\left(\frac{g(x)}{\|g\|_{L^{p(\cdot)}\left(G \backslash B\left(x_{0}, r\right)\right)}}\right)^{p(x)}\|g\|_{L^{p(\cdot)}\left(G \backslash B\left(x_{0}, r\right)\right)}^{p(x)} d x\right) \frac{d r}{r} \\
\leq & C\left(\log \frac{2 d_{G}}{t}\right)^{b p\left(x_{0}\right)} \omega\left(x_{0}, t\right)^{p\left(x_{0}\right)} \int_{0}^{t}\left(\log \frac{2 d_{G}}{r}\right)^{-b p\left(x_{0}\right)-1} \omega\left(x_{0}, r\right)^{-p^{+}\left(B\left(x_{0}, t\right)\right)} \frac{d r}{r} \leq C
\end{aligned}
$$

by (P2), Lemma 2.1 and ( $\omega 6.1)$.

Power weights can be treated simpler than Theorem 6.2 in the following manner.

Theorem 6.3. For $x_{0} \in G$, suppose

(w6.2) there exist constants $b, Q>0$ such that

$$
\int_{0}^{t} r^{b} \omega\left(x_{0}, r\right)^{-1} \frac{d r}{r} \leq Q t^{b} \omega\left(x_{0}, t\right)^{-1}
$$

for all $0<t<d_{G}$.

Then there exists a constant $C>0$ such that

$$
\int_{G}|f(x) g(x)| d x \leq C\|f\|_{\mathcal{\mathcal { H }}_{\left\{x_{0}\right\}}^{q(\cdot), 1, \eta}(G)}\|g\|_{\overline{\mathcal{H}}_{\left\{x_{0}\right\}}^{p(\cdot), \infty, \omega}(G)}
$$

for all measurable functions $f$ and $g$ on $G$, where $\eta\left(x_{0}, r\right)=\omega\left(x_{0}, r\right)^{-1}$.

Proof. Let $x_{0} \in G$. Let $f$ and $g$ be nonnegative measurable functions on $G$ such that $\|f\|_{\overline{\mathcal{H}}_{\left\{x_{0}\right\}}^{q(\cdot), 1, \eta}(G)} \leq 1$ and $\|g\|_{\underline{\mathcal{H}}_{\left\{x_{0}\right\}}^{p(\cdot), \infty, \omega}(G)} \leq 1$. For $b>0$, we have by Fubini's theorem and Hölder's inequality

$$
\begin{aligned}
\int_{G} f(x) g(x) d x & \leq C \int_{0}^{2 d_{G}}\left(\int_{B\left(x_{0}, t\right)} f(x) g(x)\left|x-x_{0}\right|^{b} d x\right) t^{-b} \frac{d t}{t} \\
& \leq C \int_{0}^{2 d_{G}}\|f\|_{L^{q(\cdot)}\left(B\left(x_{0}, t\right)\right)}\left\|g\left|\cdot-x_{0}\right|^{b}\right\|_{L^{p(\cdot)}\left(B\left(x_{0}, t\right)\right)} t^{-b} \frac{d t}{t}
\end{aligned}
$$

First, we show that

$$
\left\|g\left|\cdot-x_{0}\right|^{b}\right\|_{L^{p(\cdot)\left(B\left(x_{0}, 2 s\right) \backslash B\left(x_{0}, s\right)\right)}} \leq C s^{b} \omega\left(x_{0}, s\right)^{-1} \leq C s^{b} \eta\left(x_{0}, s\right)
$$

for all $0<s<d_{G}$. In fact, we obtain

$$
\begin{aligned}
& \int_{B\left(x_{0}, 2 s\right) \backslash B\left(x_{0}, s\right)}\left(\frac{g(x)}{s^{b} \omega\left(x_{0}, s\right)^{-1}}\right)^{p(x)}\left|x-x_{0}\right|^{p p(x)} d x \\
& \leq C \int_{B\left(x_{0}, 2 s\right) \backslash B\left(x_{0}, s\right)}\left(\frac{g(x)}{\|g\|_{L^{p(\cdot)}\left(B\left(x_{0}, 2 s\right) \backslash B\left(x_{0}, s\right)\right)}}\right)^{p(x)} \\
& \cdot\left(\omega\left(x_{0}, s\right)\|g\|_{L^{p(\cdot)}\left(B\left(x_{0}, 2 s\right) \backslash B\left(x_{0}, s\right)\right)}\right)^{p(x)} d x \leq C
\end{aligned}
$$


by (P2) and Lemma 2.1, which gives

$$
\begin{aligned}
\left\|g\left|\cdot-x_{0}\right|^{b}\right\|_{L^{p(\cdot)}\left(B\left(x_{0}, t\right)\right)} & \leq \sum_{j=1}^{\infty}\left\|g\left|\cdot-x_{0}\right|^{b}\right\|_{L^{p(\cdot)}\left(B\left(x_{0}, 2^{-j+1} t\right) \backslash B\left(x_{0}, 2^{-j} t\right)\right)} \\
& \leq C \int_{0}^{t} r^{b} \omega\left(x_{0}, r\right)^{-1} \frac{d r}{r} \leq C t^{b} \omega\left(x_{0}, t\right)^{-1}
\end{aligned}
$$

by ( $\omega 6.2)$. Thus we obtain the required result.

Theorem 6.4. Let $\eta(\cdot, \cdot) \in \Omega(G)$. For $x_{0} \in G$, suppose (w6.3) there exists a constant $Q>0$ such that

$$
\int_{t}^{2 d_{G}} \eta\left(x_{0}, r\right) \frac{d r}{r} \leq Q \omega\left(x_{0}, t\right)^{-1}
$$

for all $0<t<d_{G}$.

Then there exists a constant $C>0$ such that

$$
\|f\|_{\underline{\mathcal{H}}_{\left\{x_{0}\right\}}^{q(\cdot), \eta,}(G)} \leq C \sup _{g} \int_{G}|f(x) g(x)| d x
$$

for all measurable functions $f$ on $G$, where the supremum is taken over all measurable functions $g$ on $G$ such that $\|g\|_{X} \leq 1$ with $X=\overline{\mathcal{H}}_{\left\{x_{0}\right\}}^{p(\cdot), \infty, \omega}(G)$.

Proof. Let $x_{0} \in G$. Let $f$ be a nonnegative measurable function on $G$. To show the claim, we may assume that

$$
\sup _{g} \int_{G}|f(x) g(x)| d x \leq 1
$$

where the supremum is taken over all measurable functions $g$ on $G$ such that $\|g\|_{X} \leq$ 1. Take a compact set $K \subset G \backslash\left\{x_{0}\right\}$. Since $L^{p(\cdot)}(K)=\left\{g \chi_{K}: g \in L^{p(\cdot)}(G)\right\} \subset X$, $f \chi_{K} \in L^{q(\cdot)}(G)$, in view of [25] or [16, Theorem 3.2.13]. By $(\omega 6.3)$, we find

$$
\left\|f \chi_{K}\right\|_{\underline{\mathcal{H}}_{\left\{x_{0}\right\}}^{q(\cdot), 1, \eta}(G)}<\infty
$$

and, moreover, we have by Lemma 2.2

$$
\sum_{j \in N_{0}} \eta\left(x_{0}, 2^{-j+1} d_{G}\right) F_{j} \sim\left\|f \chi_{K}\right\|_{\underline{\mathcal{H}}_{\left\{x_{0}\right\}}^{q(\cdot), 1, \eta}(G)},
$$

where $F_{j}=\left\|f_{j}\right\|_{L^{q(\cdot)}(G)}, f_{j}=f \chi_{K \cap B\left(x_{0}, 2^{-j+1} d_{G}\right)}$ and $N_{0}$ is the set of positive integers $j$ such that $F_{j}>0$. Set

$$
g(x)=\sum_{j \in N_{0}} \eta\left(x_{0}, 2^{-j+1} d_{G}\right)\left|f_{j}(x) / F_{j}\right|^{q(x)-2} f_{j}(x) / F_{j} .
$$

Then we see that

$$
\begin{aligned}
\|g\|_{L^{p(\cdot)}\left(G \backslash B\left(x_{0}, r\right)\right)} & \leq \sum_{j \in N_{0}, 2^{-j+1} d_{G}>r} \eta\left(x_{0}, 2^{-j+1} d_{G}\right)\left\|\left|f_{j} / F_{j}\right|^{q(\cdot)-2} f_{j} / F_{j}\right\|_{L^{p(\cdot)}(G)} \\
& \leq \sum_{j \geq 1,2^{-j+1} d_{G}>r} \eta\left(x_{0}, 2^{-j+1} d_{G}\right) \leq C \omega\left(x_{0}, r\right)^{-1}
\end{aligned}
$$

for all $0<r<d_{G}$ by $(\omega 6.3)$ and hence

$$
\|g\|_{\overline{\mathcal{H}}_{\left\{x_{0}\right\}}^{p(\cdot), \omega}(G)} \leq C .
$$


Consequently it follows that

$$
\begin{aligned}
\int_{G} f(x) g(x) d x & =\sum_{j \in N_{0}} \eta\left(x_{0}, 2^{-j+1} d_{G}\right) \int_{G} f(x)\left|f_{j}(x) / F_{j}\right|^{q(x)-2} f_{j}(x) / F_{j} d x \\
& =\sum_{j \in N_{0}} \eta\left(x_{0}, 2^{-j+1} d_{G}\right) F_{j} \geq C\left\|f \chi_{K}\right\|_{\underline{\mathcal{H}}_{\left\{x_{0}\right\}}^{q(\cdot), \eta}(G)} .
\end{aligned}
$$

Hence, by the monotone convergence theorem, we have

$$
\sup _{g} \int_{G} f(x) g(x) d x \geq C\|f\|_{\underline{\mathcal{H}}_{\left\{x_{0}\right\}}^{q(\cdot), 1, \eta}(G)}
$$

which gives the required inequality.

Let $X$ be a family of measurable functions on $G$ with a norm $\|\cdot\|_{X}$. Then the associate space $X^{\prime}$ of $X$ is defined as the family of all measurable functions $f$ on $G$ such that

$$
\|f\|_{X^{\prime}}=\sup _{g \in X:\|g\|_{X} \leq 1} \int_{G}|f(x) g(x)| d x<\infty .
$$

Theorems 6.2, 6.3 and 6.4 give the following result.

Corollary 6.5. For $x_{0} \in G$, suppose $(\omega 6.1)$ and ( $\left.\omega 6.3\right)$ hold. Then

$$
\left(\overline{\mathcal{H}}_{\left\{x_{0}\right\}}^{p(\cdot), \infty, \omega}(G)\right)^{\prime}=\underline{\mathcal{H}}_{\left\{x_{0}\right\}}^{q(\cdot), 1, \eta}(G),
$$

where $\eta\left(x_{0}, r\right)=\left(\log \frac{2 d_{G}}{r}\right)^{-1} \omega\left(x_{0}, r\right)^{-1}$. If $(\omega 6.2)$ and $(\omega 6.3)$ hold, then the same conclusion is fulfilled with $\eta\left(x_{0}, r\right)=\omega\left(x_{0}, r\right)^{-1}$.

For $0<q \leq \infty$, set

$$
\widetilde{\mathcal{H}}^{p(\cdot), q, \omega}(G)=\sum_{x_{0} \in G} \overline{\mathcal{H}}_{\left\{x_{0}\right\}}^{p(\cdot), q, \omega}(G),
$$

whose quasi-norm is defined by

$$
\|f\|_{\widetilde{\mathcal{H}}^{p(\cdot), q, \omega}(G)}=\inf _{|f|=\sum_{j}\left|f_{j}\right|,\left\{x_{j}\right\} \subset G} \sum_{j}\left\|f_{j}\right\|_{\overline{\mathcal{H}}_{\left\{x_{j}\right\}}^{p(\cdot), q, \omega}(G)} .
$$

The Hölder type inequality in Theorem 6.2 or 6.3 , under the same assumptions, implies

$$
\int_{G}|f(x) g(x)| d x=\sum_{j} \int_{G}\left|f(x) g_{j}(x)\right| d x \leq C\|f\|_{\underline{\mathcal{H}}^{q(\cdot), 1, \eta}(G)} \sum_{j}\left\|g_{j}\right\|_{\overline{\mathcal{H}}_{\left\{x_{j}\right\}}^{p(\cdot), \omega}(G)},
$$

so that

$$
\int_{G}|f(x) g(x)| d x \leq C\|f\|_{\underline{\mathcal{H}}^{q(\cdot), 1, \eta}(G)}\|g\|_{\widetilde{\mathcal{H}}^{p(\cdot), \infty, \omega}(G)} .
$$

Theorem 6.4 gives the converse inequality.

Theorems 6.2, 6.3 and 6.4 give the following result.

Corollary 6.6. If ( $\omega 6.1)$ and ( $\omega 6.3)$ hold for all $x_{0} \in G$ with the same constant $Q$, then

$$
\left(\widetilde{\mathcal{H}}^{p(\cdot), \infty, \omega}(G)\right)^{\prime}=\underline{\mathcal{H}}^{q(\cdot), 1, \eta}(G),
$$

where $\eta\left(x_{0}, r\right)=\left(\log \frac{2 d_{G}}{r}\right)^{-1} \omega\left(x_{0}, r\right)^{-1}$. If ( $\left.\omega 6.2\right)$ and $(\omega 6.3)$ hold for all $x_{0} \in G$ with the same constant $Q$, then the same conclusion is fulfilled with $\eta\left(x_{0}, r\right)=\omega\left(x_{0}, r\right)^{-1}$. 
Remark 6.7. For $0<q \leq \infty$, set

$$
\overline{\mathcal{H}}^{p(\cdot), q, \omega}(G)=\bigcap_{x_{0} \in G} \overline{\mathcal{H}}_{\left\{x_{0}\right\}}^{p(\cdot), \omega}(G)
$$

and define the norm

$$
\|f\|_{\overline{\mathcal{H}}^{p(\cdot), q, \omega}(G)}=\sup _{x_{0} \in G}\|f\|_{\overline{\mathcal{H}}_{\left\{x_{0}\right\}}^{p(\cdot), \omega}(G)},
$$

as usual. Then note that

$$
\overline{\mathcal{H}}^{p(\cdot), \infty, \omega}(G)= \begin{cases}L^{p(\cdot)}(G), & \omega(x, 0)=0 \text { for all } x \in G ; \\ \{0\}, & \omega(x, 0)=\infty \text { for all } x \in G .\end{cases}
$$

For related results, we refer the reader to the paper by Di Fratta and Fiorenza [17] with logarithmic weights, and the paper by Gagatishvili and Mustafayev [19] with general weights.

Remark 6.8. If $\omega(t)=\left(\log \left(2 d_{G} / t\right)\right)^{-a}$ with $a>0$, then $(\omega 6.1)$ and $(\omega 6.3)$ hold for $\eta(t)=\left(\log \left(2 d_{G} / t\right)\right)^{a-1}$; and if $\omega(t)=r^{a}$ with $a>0$, then $(\omega 6.2)$ and $(\omega 6.3)$ hold for $\eta(t)=t^{-a}$.

\section{Associate spaces of $\underline{\mathcal{H}}_{\left\{x_{0}\right\}}^{p(\cdot), \infty, \omega}(G)$}

Recall that for $x_{0} \in G$ and measurable functions $f$ on $G$,

$$
\|f\|_{\underline{\mathcal{H}}_{\left\{x_{0}\right\}}^{p(\cdot), \infty, \omega}(G)}=\sup _{0<t<d_{G}} \omega\left(x_{0}, t\right)\|f\|_{L^{p(\cdot)}\left(B\left(x_{0}, t\right)\right)}
$$

and

$$
\|f\|_{\overline{\mathcal{H}}_{\left\{x_{0}\right\}}^{p(\cdot), 1, \omega}(G)}=\int_{0}^{2 d_{G}} \omega\left(x_{0}, t\right)\|f\|_{L^{p(\cdot)}\left(G \backslash B\left(x_{0}, t\right)\right)} \frac{d t}{t} .
$$

We have the Hölder type inequality for log type weights $\omega$.

Theorem 7.1. For $x_{0} \in G$, suppose

( $\omega 7.1)$ there exist constants $r_{0}, b, Q>0$ such that

$$
\begin{aligned}
& \int_{t}^{2 r_{0}}\left(\left(\log \frac{2 d_{G}}{t}\right)^{b} \omega\left(x_{0}, t\right)^{-1}\right)^{c_{p} / \log \left(2 d_{G} / r\right)}\left(\left(\log \frac{2 d_{G}}{r}\right)^{b} \omega\left(x_{0}, r\right)^{-1}\right)^{p\left(x_{0}\right)} \\
& \cdot\left(\log \frac{2 d_{G}}{r}\right)^{-1} \frac{d r}{r} \leq Q\left(\left(\log \frac{2 d_{G}}{t}\right)^{b} \omega\left(x_{0}, t\right)^{-1}\right)^{p\left(x_{0}\right)}
\end{aligned}
$$

for all $0<t<r_{0}$.

Then there exists a constant $C>0$ such that

$$
\int_{G}|f(x) g(x)| d x \leq C\|f\|_{\overline{\mathcal{H}}_{\left\{x_{0}\right\}}^{q(\cdot), 1, \eta}(G)}\|g\|_{\mathcal{\mathcal { H }}_{\left\{x_{0}\right\}}^{p(\cdot), \infty, \omega}(G)}
$$

for all measurable functions $f, g$ on $G$, where $\eta\left(x_{0}, r\right)=\left(\log \frac{2 d_{G}}{r}\right)^{-1} \omega\left(x_{0}, r\right)^{-1}$.

Proof. Let $x_{0} \in G$. Let $f$ and $g$ be nonnegative measurable functions on $G$ such that $\|f\|_{\overline{\mathcal{H}}_{\left\{x_{0}\right\}}^{q(\cdot), 1, \eta}(G)} \leq 1$ and $\|g\|_{\underline{\mathcal{H}}_{\left\{x_{0}\right\}}^{p(\cdot), \infty, \omega}(G)} \leq 1$. For $b>0$ we have by Fubini's theorem 
and Hölder's inequality

$$
\begin{aligned}
& \int_{G} f(x) g(x) d x \\
& \leq C \int_{0}^{d_{G}}\|f\|_{L^{q(\cdot)}\left(G \backslash B\left(x_{0}, t\right)\right)}\left\|g\left(\log \frac{2 d_{G}}{\left|\cdot-x_{0}\right|}\right)^{b}\right\|_{L^{p(\cdot)}\left(G \backslash B\left(x_{0}, t\right)\right)}\left(\log \frac{2 d_{G}}{t}\right)^{-b-1} \frac{d t}{t}
\end{aligned}
$$

as in the proof of Theorem 6.2. It suffices to show

$$
\begin{aligned}
\left\|g\left(\log \frac{2 d_{G}}{\left|\cdot-x_{0}\right|}\right)^{b}\right\|_{L^{p(\cdot)}\left(G \backslash B\left(x_{0}, t\right)\right)} & \leq C\left(\log \frac{2 d_{G}}{t}\right)^{b} \omega\left(x_{0}, t\right)^{-1} \\
& =C\left(\log \frac{2 d_{G}}{t}\right)^{b+1} \eta\left(x_{0}, t\right)
\end{aligned}
$$

for all $0<t<d_{G}$. In fact, we obtain for $0<r_{0}<d_{G}$

$$
\begin{aligned}
& \int_{B\left(x_{0}, r_{0}\right) \backslash B\left(x_{0}, t\right)}\left(\frac{g(x)}{\left(\log \left(2 d_{G} / t\right)\right)^{b} \omega\left(x_{0}, t\right)^{-1}}\right)^{p(x)}\left(\log \frac{2 d_{G}}{\left|x-x_{0}\right|}\right)^{b p(x)} d x \\
& \leq C \int_{B\left(x_{0}, r_{0}\right) \backslash B\left(x_{0}, t\right)}\left(\frac{g(x)}{\left(\log \left(2 d_{G} / t\right)\right)^{b} \omega\left(x_{0}, t\right)^{-1}}\right)^{p(x)}\left(\log \frac{2 d_{G}}{\left|x-x_{0}\right|}\right)^{b p\left(x_{0}\right)} d x \\
& \leq C \int_{B\left(x_{0}, r_{0}\right) \backslash B\left(x_{0}, t\right)}\left(\frac{g(x)}{\left(\log \left(2 d_{G} / t\right)\right)^{b} \omega\left(x_{0}, t\right)^{-1}}\right)^{p(x)}\left(\int_{\left|x-x_{0}\right|}^{2 r_{0}}\left(\log \frac{2 d_{G}}{r}\right)^{b p\left(x_{0}\right)-1} \frac{d r}{r}\right) d x \\
& \leq C \int_{t}^{2 r_{0}}\left(\int_{B\left(x_{0}, r\right) \backslash B\left(x_{0}, t\right)} g(x)^{p(x)}\left(\left(\log \frac{2 d_{G}}{t}\right)^{-b} \omega\left(x_{0}, t\right)\right)^{p(x)}\right. \\
& \left.\cdot\left(\log \frac{2 d_{G}}{r}\right)^{b p\left(x_{0}\right)-1} d x\right) \frac{d r}{r} \\
& \leq C\left(\log \frac{2 d_{G}}{t}\right)^{-b p\left(x_{0}\right)} \omega\left(x_{0}, t\right)^{p\left(x_{0}\right)} \int_{t}^{2 r_{0}}\left(\left(\log \frac{2 d_{G}}{t}\right)^{b} \omega\left(x_{0}, t\right)^{-1}\right)^{c_{p} / \log \left(2 d_{G} / r\right)} \\
& \cdot\left(\log \frac{2 d_{G}}{r}\right)^{b p\left(x_{0}\right)-1}\left(\int_{B\left(x_{0}, r\right)} g(x)^{p(x)} d x\right) \frac{d r}{r} \\
& \leq C\left(\log \frac{2 d_{G}}{t}\right)^{-b p\left(x_{0}\right)} \omega\left(x_{0}, t\right)^{p\left(x_{0}\right)} \int_{t}^{2 r_{0}}\left(\left(\log \frac{2 d_{G}}{t}\right)^{b} \omega\left(x_{0}, t\right)^{-1}\right)^{c_{p} / \log \left(2 d_{G} / r\right)} \\
& \cdot\left(\left(\log \frac{2 d_{G}}{r}\right)^{b} \omega\left(x_{0}, r\right)^{-1}\right)^{p\left(x_{0}\right)}\left(\log \frac{2 d_{G}}{r}\right)^{-1} \frac{d r}{r} \\
& \leq C\left(\log \frac{2 d_{G}}{t}\right)^{-b p\left(x_{0}\right)} \omega\left(x_{0}, t\right)^{p\left(x_{0}\right)}\left(\left(\log \frac{2 d_{G}}{t}\right)^{b} \omega\left(x_{0}, t\right)^{-1}\right)^{p\left(x_{0}\right)} \leq C
\end{aligned}
$$


by (P2), condition ( $\omega 7.1)$ and Lemmas 2.1 and 2.4 , which gives

$$
\left\|g\left(\log \frac{2 d_{G}}{\left|\cdot-x_{0}\right|}\right)^{b}\right\|_{L^{p(\cdot)\left(B\left(x_{0}, r_{0}\right) \backslash B\left(x_{0}, t\right)\right)}} \leq C\left(\log \frac{2 d_{G}}{t}\right)^{b} \omega\left(x_{0}, t\right)^{-1}
$$

for all $0<t<r_{0}$. Moreover,

$$
\left\|g\left(\log \frac{2 d_{G}}{\left|\cdot-x_{0}\right|}\right)^{b}\right\|_{L^{p(\cdot)}\left(G \backslash B\left(x_{0}, r_{0}\right)\right)} \leq C\|g\|_{L^{p(\cdot)}\left(G \backslash B\left(x_{0}, r_{0}\right)\right)} \leq C,
$$

which completes the proof.

Remark 7.2. We show that $\omega(t)=\left(\log \left(2 d_{G} / t\right)\right)^{a}$ with $a>0$ satisfies $(\omega 7.1)$. To show this, for $b, c>0$ one can find constants $r_{0}, Q>0$ such that

$$
\int_{t}^{2 r_{0}}\left(\log \frac{2 d_{G}}{t}\right)^{c / \log \left(2 d_{G} / r\right)}\left(\log \frac{2 d_{G}}{r}\right)^{b-1} \frac{d r}{r} \leq Q\left(\log \frac{2 d_{G}}{t}\right)^{b}
$$

for all $0<t<r_{0}$ and $x_{0} \in G$. In fact, first find $0<r_{0}<d_{G} / e$ such that $\varepsilon=$ $1 / \log \left(d_{G} / r_{0}\right)<b / 2 c$, and note for $\tilde{t}=2 d_{G} e^{-\left(\log \left(2 d_{G} / t\right)\right)^{1 / 2}}$ that

$$
\begin{aligned}
\int_{t}^{\tilde{t}}\left(\log \frac{2 d_{G}}{t}\right)^{c / \log \left(2 d_{G} / r\right)}\left(\log \frac{2 d_{G}}{r}\right)^{b-1} \frac{d r}{r} & \leq C \int_{t}^{2 d_{G}}\left(\log \frac{2 d_{G}}{r}\right)^{b-1} \frac{d r}{r} \\
& \leq Q\left(\log \frac{2 d_{G}}{t}\right)^{b}
\end{aligned}
$$

since $\left(\log \left(2 d_{G} / t\right)\right)^{c / \log \left(2 d_{G} / r\right)} \leq C$ for all $t<r<\tilde{t}$ and

$$
\begin{aligned}
\int_{\tilde{t}}^{2 r_{0}}\left(\log \frac{2 d_{G}}{t}\right)^{c / \log \left(2 d_{G} / r\right)}\left(\log \frac{2 d_{G}}{r}\right)^{b-1} \frac{d r}{r} & \leq C\left(\log \frac{2 d_{G}}{t}\right)^{c \varepsilon} \int_{\tilde{t}}^{2 r_{0}}\left(\log \frac{2 d_{G}}{r}\right)^{b-1} \frac{d r}{r} \\
& \leq Q\left(\log \frac{2 d_{G}}{t}\right)^{c \varepsilon+b / 2} \leq Q\left(\log \frac{2 d_{G}}{t}\right)^{b}
\end{aligned}
$$

as required.

For power weights $\omega$, we obtain the following result.

Theorem 7.3. For $x_{0} \in G$, suppose

( $\omega 7.2)$ there exist constants $b, Q>0$ such that

$$
\int_{t}^{2 d_{G}} r^{-b} \omega\left(x_{0}, r\right)^{-1} \frac{d r}{r} \leq Q r^{-b} \omega\left(x_{0}, t\right)^{-1}
$$

for all $0<t<d_{G}$.

Then there exists a constant $C>0$ such that

$$
\int_{G}|f(x) g(x)| d x \leq C\|f\|_{\overline{\mathcal{H}}_{\left\{x_{0}\right\}}^{q \cdot \cdot, 1, \eta}(G)}\|g\|_{\underline{\mathcal{H}}_{\left\{x_{0}\right\}}^{p(\cdot), \infty, \omega}(G)}
$$

for all measurable functions $f, g$ on $G$, where $\eta\left(x_{0}, r\right)=\omega\left(x_{0}, r\right)^{-1}$.

As in the proof of Theorem 6.4, we have the following result.

Theorem 7.4. Let $\eta(\cdot, \cdot) \in \Omega(G)$. For $x_{0} \in G$, suppose 
( $\omega 7.3)$ there exists a constant $Q>0$ such that

$$
\int_{0}^{t} \eta\left(x_{0}, r\right) \frac{d r}{r} \leq Q \omega\left(x_{0}, t\right)^{-1}
$$

for all $0<t<d_{G}$.

Then there exists a constant $C>0$ such that

$$
\|f\|_{\overline{\mathcal{H}}_{q(\cdot), 1, \eta}^{\left\{x_{0}\right\}}(G)} \leq C \sup _{g} \int_{G}|f(x) g(x)| d x
$$

for all measurable functions $f$ on $G$, where the supremum is taken over all measurable functions $g$ on $G$ such that $\|g\|_{X} \leq 1$ with $X=\underline{\mathcal{H}}_{\left\{x_{0}\right\}}^{p(\cdot), \omega}(G)$.

Theorems 7.1, 7.3 and 7.4 give the following result.

Corollary 7.5. If ( $\omega 7.1)$ and ( $\omega 7.3)$ hold for $x_{0} \in G$, then

$$
\left(\underline{\mathcal{H}}_{\left\{x_{0}\right\}}^{p(\cdot), \omega}(G)\right)^{\prime}=\overline{\mathcal{H}}_{\left\{x_{0}\right\}}^{q(\cdot), 1, \eta}(G),
$$

where $\eta\left(x_{0}, r\right)=\left(\log \frac{2 d_{G}}{r}\right)^{-1} \omega\left(x_{0}, r\right)^{-1}$. If $(\omega 7.2)$ and $(\omega 7.3)$ hold for $x_{0} \in G$, then the same conclusion is fulfilled with $\eta\left(x_{0}, r\right)=\omega\left(x_{0}, r\right)^{-1}$.

Remark 7.6. If $\omega(t)=\left(\log \left(2 d_{G} / t\right)\right)^{a}$ with $a>0$, then $(\omega 7.1)$ and $(\omega 7.3)$ hold for $\eta(t)=\left(\log \left(2 d_{G} / t\right)\right)^{-a-1}$; and if $\omega(t)=t^{-a}$ with $a>0$, then $(\omega 7.2)$ and $(\omega 7.3)$ hold for $\eta(t)=t^{a}$.

For $0<q \leq \infty$, we may consider

$$
{\underset{\sim}{\mathcal{H}}}^{p(\cdot), q, \omega}(G)=\sum_{x_{0} \in G} \underline{\mathcal{H}}_{\left\{x_{0}\right\}}^{p(\cdot), q, \omega}(G),
$$

whose quasi-norm is defined by

$$
\|f\|_{\mathcal{H}^{p(\cdot), q, \omega}(G)}=\inf _{|f|=\sum_{j}\left|f_{j}\right|,\left\{x_{j}\right\} \subset G} \sum_{j}\left\|f_{j}\right\|_{\underline{\mathcal{H}}_{\left\{x_{j}\right\}}^{p(\cdot, q, \omega}(G)} .
$$

One can show that

$$
\underset{\sim}{\mathcal{H}^{p(\cdot), q, \omega}}(G)=L^{p(\cdot)}(G) .
$$

For this, we only show the inclusion $L^{p(\cdot)}(G) \subset \underset{\sim}{\mathcal{H}^{p(\cdot), q, \omega}}(G)$. Take $f \in L^{p(\cdot)}(G)$ and $x_{1}, x_{2} \in G\left(x_{1} \neq x_{2}\right)$. Write

$$
f=f \chi_{B\left(x_{2},\left|x_{1}-x_{2}\right| / 2\right)}+f \chi_{G \backslash B\left(x_{2},\left|x_{1}-x_{2}\right| / 2\right)}=f_{1}+f_{2} .
$$

Then

$$
\begin{aligned}
\left\|f_{1}\right\|_{\underline{\mathcal{H}}_{\left\{x_{1}\right\}}^{p(\cdot), \omega}(G)} & \leq\left(\int_{\left|x_{1}-x_{2}\right| / 2}^{2 d_{G}}\left(\omega\left(x_{1}, r\right)\left\|f_{1}\right\|_{L^{p(\cdot)}\left(B\left(x_{1}, r\right)\right)}\right)^{q} d r / r\right)^{1 / q} \\
& \leq\left\|f_{1}\right\|_{L^{p(\cdot)}(G)}\left(\int_{\left|x_{1}-x_{2}\right| / 2}^{2 d_{G}} \omega\left(x_{1}, r\right)^{q} d r / r\right)^{1 / q}=A\left\|f_{1}\right\|_{L^{p(\cdot)}(G)}
\end{aligned}
$$


and

$$
\begin{aligned}
\left\|f_{2}\right\|_{\underline{\mathcal{H}}_{\left\{x_{2}\right\}}^{p(\cdot,), \omega}(G)} & \leq\left(\int_{\left|x_{1}-x_{2}\right| / 2}^{2 d_{G}}\left(\omega\left(x_{2}, r\right)\left\|f_{2}\right\|_{L^{p(\cdot)}\left(B\left(x_{2}, r\right)\right)}\right)^{q} d r / r\right)^{1 / q} \\
& \leq\left\|f_{2}\right\|_{L^{p(\cdot)}(G)}\left(\int_{\left|x_{1}-x_{2}\right| / 2}^{2 d_{G}} \omega\left(x_{2}, r\right)^{q} d r / r\right)^{1 / q}=B\left\|f_{2}\right\|_{L^{p(\cdot)}(G)} .
\end{aligned}
$$

Hence

$$
\begin{aligned}
\|f\|_{\mathcal{H}^{p(\cdot), q, \omega}(G)} & \leq\left\|f_{1}\right\|_{\underline{\mathcal{H}}_{\left\{x_{1}\right\}}^{p(\cdot,)}(G)}+\left\|f_{2}\right\|_{\underline{\mathcal{H}}_{\left\{x_{2}\right\}}^{p(\cdot), q}(G)} \leq A\left\|f_{1}\right\|_{L^{p(\cdot)}(G)}+B\left\|f_{2}\right\|_{L^{p(\cdot)}(G)} \\
& \leq(A+B)\|f\|_{L^{p(\cdot)}(G)}<\infty,
\end{aligned}
$$

as required.

\section{Associate spaces of $\underline{\mathcal{H}}_{\left\{x_{0}\right\}}^{p(\cdot), 1, \omega}(G)$}

Theorem 8.1. Let $\eta(\cdot, \cdot) \in \Omega(G), x_{0} \in G$ and $X=\underline{\mathcal{H}}_{\left\{x_{0}\right\}}^{p(), 1, \omega}(G)$. Suppose (w8.1) there exists a constant $Q>0$ such that

$$
\int_{t}^{2 d_{G}} \omega\left(x_{0}, r\right) \frac{d r}{r} \leq Q \eta\left(x_{0}, t\right)^{-1}
$$

for all $0<t<d_{G}$.

Then there exists a constant $C>0$ such that

$$
\|f\|_{\overline{\mathcal{H}}_{\left\{x_{0}\right\}}^{q(\cdot), \infty, \eta}(G)} \leq C\|f\|_{X^{\prime}}
$$

for all measurable functions $f$ on $G$.

Proof. Let $x_{0} \in G$. First we show

$$
\int_{G \backslash B\left(x_{0}, R\right)} f(x) g(x) d x \leq C \eta\left(x_{0}, R\right)^{-1}\|g\|_{L^{p(\cdot)}\left(G \backslash B\left(x_{0}, R\right)\right)}\|f\|_{X^{\prime}}
$$

for $0<R<d_{G}$ and nonnegative measurable functions $f, g$ on $G$. To show this, we consider

$$
h=\eta\left(x_{0}, R\right) g \chi_{G \backslash B\left(x_{0}, R\right)} /\|g\|_{L^{p(\cdot)}\left(G \backslash B\left(x_{0}, R\right)\right)}
$$

when $0<\|g\|_{L^{p(\cdot)}\left(G \backslash B\left(x_{0}, R\right)\right)}<\infty$. Then we have by $(\omega 8.1)$

$$
\int_{0}^{2 d_{G}} \omega\left(x_{0}, t\right)\|h\|_{L^{p(\cdot)}\left(B\left(x_{0}, t\right)\right)} \frac{d t}{t} \leq \eta\left(x_{0}, R\right) \int_{R}^{2 d_{G}} \omega\left(x_{0}, t\right) \frac{d t}{t} \leq C,
$$

and hence

$$
\int_{G \backslash B\left(x_{0}, R\right)} f(x) h(x) d x \leq C\|f\|_{X^{\prime}}
$$

Now we obtain

$$
\int_{G \backslash B\left(x_{0}, R\right)} f(x) g(x) d x \leq C \eta\left(x_{0}, R\right)^{-1}\|g\|_{L^{p(\cdot)}\left(G \backslash B\left(x_{0}, R\right)\right)}\|f\|_{X^{\prime}} .
$$

If we take $g(x)=\left|f(x) /\|f\|_{L^{q(\cdot)}\left(G \backslash B\left(x_{0}, R\right)\right)}\right|^{q(x)-1} \chi_{G \backslash B\left(x_{0}, R\right)}$ when $0<\|f\|_{L^{q(\cdot)}\left(G \backslash B\left(x_{0}, R\right)\right)}<$ $\infty$, then we have by (8.1) that 


$$
\begin{aligned}
1 & =\int_{G \backslash B\left(x_{0}, R\right)}\left\{f(x) /\|f\|_{L^{q(\cdot)}\left(G \backslash B\left(x_{0}, R\right)\right)}\right\}^{q(x)} d x \\
& \leq C \eta\left(x_{0}, R\right)^{-1}\left\|\left\{f /\|f\|_{L^{q(\cdot)}\left(G \backslash B\left(x_{0}, R\right)\right)}\right\}^{q(\cdot)-1}\right\|_{L^{p(\cdot)}\left(G \backslash B\left(x_{0}, R\right)\right)}\|f /\| f\left\|_{L^{q(\cdot)}\left(G \backslash B\left(x_{0}, R\right)\right)}\right\|_{X^{\prime}} \\
& \leq C \eta\left(x_{0}, R\right)^{-1}\left\{\|f\|_{L^{q(\cdot)}\left(G \backslash B\left(x_{0}, R\right)\right)}\right\}^{-1}\|f\|_{X^{\prime}},
\end{aligned}
$$

which shows

$$
\eta\left(x_{0}, R\right)\|f\|_{L^{q(\cdot)}\left(G \backslash B\left(x_{0}, R\right)\right)} \leq C\|f\|_{X^{\prime}}
$$

Thus it follows that

$$
\|f\|_{\overline{\mathcal{H}}_{\left\{x_{0}\right\}}^{q(\cdot), \infty, \eta}(G)} \leq C\|f\|_{X^{\prime}}
$$

as required.

Corollary 8.2. If ( $\omega 8.1)$ holds for $x_{0} \in G$ and ( $\left.\omega 6.1\right)$ holds for $x_{0} \in G, \eta$ and $q(\cdot)$, then

$$
\left(\underline{\mathcal{H}}_{\left\{x_{0}\right\}}^{p(\cdot), 1, \omega}(G)\right)^{\prime}=\overline{\mathcal{H}}_{\left\{x_{0}\right\}}^{q(\cdot), \infty, \eta}(G),
$$

where $\eta\left(x_{0}, r\right)=\left(\log \frac{2 d_{G}}{r}\right)^{-1} \omega\left(x_{0}, r\right)^{-1}$. If (w8.1) holds for $x_{0} \in G$ and $(\omega 6.2)$ holds for $x_{0} \in G, \eta$ and $q(\cdot)$, then the same conclusion is fulfilled with $\eta\left(x_{0}, r\right)=\omega\left(x_{0}, r\right)^{-1}$.

As in Fiorenza-Rakotoson [18, Corollary 1], we see that the associate and dual spaces of $\underline{\mathcal{H}}_{\left\{x_{0}\right\}}^{p(\cdot), \omega}(G)$ coincides with each other.

Remark 8.3. If $\omega(t)=\left(\log \left(2 d_{G} / t\right)\right)^{-1 / a}$ with $a>1$, then ( $\left.\omega 8.1\right)$ holds for $\eta(t)=\left(\log \left(2 d_{G} / t\right)\right)^{-1 / a^{\prime}}$; and if $\omega(t)=t^{-a}$ with $a>0$, then $(\omega 8.1)$ holds for $\eta(t)=t^{a}$.

\section{Associate space of $\overline{\mathcal{H}}_{\left\{x_{0}\right\}}^{p(\cdot), 1, \omega}(G)$}

As in the proof of Theorem 8.1, we have the following result.

Theorem 9.1. Let $\eta(\cdot, \cdot) \in \Omega(G), x_{0} \in G$ and $X=\overline{\mathcal{H}}_{\left\{x_{0}\right\}}^{p(\cdot), \omega}(G)$. Suppose ( $\omega 9.1)$ there exists a constant $Q>0$ such that

$$
\int_{0}^{t} \omega\left(x_{0}, r\right) \frac{d r}{r} \leq Q \eta\left(x_{0}, t\right)^{-1}
$$

for all $0<t<d_{G}$.

Then there exists a constant $C>0$ such that

$$
\|f\|_{\underline{\mathcal{H}}_{\left\{x_{0}\right\}}^{q(\cdot), \infty, \eta}(G)} \leq C\|f\|_{X^{\prime}}
$$

for all measurable functions $f$ on $G$.

Corollary 9.2. If ( $\omega 9.1)$ holds for $x_{0} \in G$ and ( $\left.\omega 7.1\right)$ holds for $x_{0} \in G, \eta$ and $q(\cdot)$, then

$$
\left(\overline{\mathcal{H}}_{\left\{x_{0}\right\}}^{p(\cdot), 1, \omega}(G)\right)^{\prime}=\underline{\mathcal{H}}_{\left\{x_{0}\right\}}^{q(\cdot), \infty, \eta}(G),
$$

where $\eta\left(x_{0}, r\right)=\left(\log \frac{2 d_{G}}{r}\right)^{-1} \omega\left(x_{0}, r\right)^{-1}$. If $(\omega 9.1)$ holds for $x_{0} \in G$ and $(\omega 7.2)$ holds for $x_{0} \in G, \eta$ and $q(\cdot)$, then the same conclusion is fulfilled with $\eta\left(x_{0}, r\right)=\omega\left(x_{0}, r\right)^{-1}$. 
Corollary 9.3. If ( $\omega 9.1)$ holds for all $x_{0} \in G$ with the same constant $Q$ and $(\omega 7.1)$ holds for $\eta, q(\cdot)$ and all $x_{0} \in G$ with the same constant $Q$, then

$$
\left(\widetilde{\mathcal{H}}^{p(\cdot), 1, \omega}(G)\right)^{\prime}=\underline{\mathcal{H}}^{q(\cdot), \infty, \eta}(G),
$$

where $\eta\left(x_{0}, r\right)=\left(\log \frac{2 d_{G}}{r}\right)^{-1} \omega\left(x_{0}, r\right)^{-1}$. If $(\omega 9.1)$ holds for all $x_{0} \in G$ with the same constant $Q$ and $(\omega 7.2)$ holds for $\eta, q(\cdot)$ and all $x_{0} \in G$ with the same constant $Q$, then the same conclusion is fulfilled with $\eta\left(x_{0}, r\right)=\omega\left(x_{0}, r\right)^{-1}$.

This corollary gives a characterization of Morrey spaces of variable exponents; see also the paper by Gogatishvili and Mustafayev [19] for constant exponents.

Remark 9.4. If $\omega(t)=\left(\log \left(2 d_{G} / t\right)\right)^{-a-1}$ with $a>0$, then $(\omega 9.1)$ holds for $\eta(t)=\left(\log \left(2 d_{G} / t\right)\right)^{a}$; and if $\omega(t)=t^{a}$ with $a>0$, then $(\omega 9.1)$ holds for $\eta(t)=t^{-a}$.

\section{Grand and small Lebesgue spaces}

Following Capone-Fiorenza [11], for $0<\theta<1$ and measurable functions $f$ on the unit ball $\mathbf{B}=B(0,1)$, we define the norm

$$
\|f\|_{\overline{\mathcal{H}}_{\{0\}}^{p(\cdot), \infty, \theta}(\mathbf{B})}=\sup _{0<t<1}\left(\log \frac{2}{t}\right)^{-\theta / p(0)}\|f\|_{L^{p(\cdot)}(\mathbf{B} \backslash B(0, t))}
$$

and

$$
\|f\|_{L^{p(\cdot)-0, \theta}(\mathbf{B})}=\sup _{0<\varepsilon<p^{-}-1} \varepsilon^{\theta / p(0)}\|f\|_{L^{p(\cdot)-\varepsilon}(\mathbf{B})} .
$$

Theorem 10.1. There exists a constant $C>0$ such that

$$
\|f\|_{L^{p(\cdot)-0, \theta}(\mathbf{B})} \leq C\|f\|_{\overline{\mathcal{H}}_{\{0\}}^{p(\cdot), \infty, \theta}(\mathbf{B})}
$$

for all measurable functions $f$ on $\mathbf{B}$.

Proof. Let $f$ be a nonnegative measurable function on $\mathbf{B}$ such that $\|f\|_{\overline{\mathcal{H}}_{\{0\}}^{p(\cdot), \infty, \theta}(\mathbf{B})} \leq$ 1 or

$$
\int_{\mathbf{B} \backslash B(0, t)}\left(\left(\log \frac{2}{t}\right)^{-\theta / p(0)} f(x)\right)^{p(x)} d x \leq 1
$$

for all $0<t<1$. For $0<\varepsilon<p^{-}-1$, we take $0<s<1$ such that $\varepsilon=\left(p^{-}-\right.$ 1) $(\log 2) / \log (2 / s)$. We have

$$
\int_{\mathbf{B} \backslash B(0, s)}\left(\varepsilon^{\theta / p(0)} f(x)\right)^{p(x)-\varepsilon} d x \leq \int_{\mathbf{B} \backslash B(0, s)} 1 d x+\int_{\mathbf{B} \backslash B(0, s)}\left(\varepsilon^{\theta / p(0)} f(x)\right)^{p(x)} d x \leq C .
$$

By multiplying (10.1) by $(\log (2 / t))^{-b-1}$ for (large) $b>1$, integration gives

$$
\begin{aligned}
& \int_{0}^{r}\left(\log \frac{2}{t}\right)^{-b-1} \frac{d t}{t} \\
& \geq \int_{0}^{r}\left(\log \frac{2}{t}\right)^{-b-1}\left(\int_{B(0, r) \backslash B(0, t)}\left(\left(\log \frac{2}{t}\right)^{-\theta / p(0)} f(x)\right)^{p(x)} d x\right) \frac{d t}{t}
\end{aligned}
$$




$$
\begin{aligned}
& \geq \int_{0}^{r}\left(\log \frac{2}{t}\right)^{-b-1}\left(\int_{B(0, r) \backslash B(0, t)}\left(\log \frac{2}{t}\right)^{-\theta-c_{p} / \log (2 /|x|)} f(x)^{p(x)} d x\right) \frac{d t}{t} \\
& =\int_{B(0, r)} f(x)^{p(x)}\left(\int_{0}^{|x|}\left(\log \frac{2}{t}\right)^{-b-1-\theta-c_{p} / \log (2 /|x|)} \frac{d t}{t}\right) d x \\
& \geq C \int_{B(0, r)} f(x)^{p(x)}\left(\log \frac{2}{|x|}\right)^{-b-\theta} d x,
\end{aligned}
$$

or

$$
\int_{B(0, r)} f(x)^{p(x)}\left(\log \frac{2}{|x|}\right)^{-b-\theta} d x \leq C\left(\log \frac{2}{r}\right)^{-b}
$$

for $0<r<1$.

First consider the case when

$$
A=\int_{B(0, s)}\left(\log \frac{2}{|x|}\right)^{(p(0)-\varepsilon)(\theta+b) / \varepsilon} d x \geq 1 .
$$

For $k>1$, we obtain

$$
\begin{aligned}
& \int_{B(0, s)}\left(\varepsilon^{\theta / p(0)} f(x)\right)^{p(x)-\varepsilon} d x \\
& \leq \int_{B(0, s)}\left(\varepsilon^{k} A^{-1 / p(0)}\left(\log \frac{2}{|x|}\right)^{(\theta+b) / \varepsilon}\right)^{p(x)-\varepsilon} d x \\
& \quad+\int_{B(0, s)}\left(\varepsilon^{\theta / p(0)} f(x)\right)^{p(x)-\varepsilon}\left(\frac{\varepsilon^{\theta / p(0)} f(x)}{\varepsilon^{k} A^{-1 / p(0)}(\log (2 /|x|))^{(\theta+b) / \varepsilon}}\right)^{\varepsilon} d x \\
& \leq C\left\{\varepsilon^{k p(0)} \int_{B(0, s)} A^{-(p(x)-\varepsilon) / p(0)}\left(\log \frac{2}{|x|}\right)^{(p(0)-\varepsilon)(\theta+b) / \varepsilon} d x\right. \\
& \left.\quad+\varepsilon^{\theta} A^{\varepsilon / p(0)} \int_{B(0, s)} f(x)^{p(x)}\left(\log \frac{2}{|x|}\right)^{-(\theta+b)} d x\right\}
\end{aligned}
$$

since $\varepsilon^{p(x)-\varepsilon} \leq C \varepsilon^{p(0)}$ by (P2) for all $x \in B(0, s)$. Since $\log (2 / t) \leq\left(2^{a} / a\right) t^{-a}$ for $0<t<1$ and $a=\varepsilon /\{2(p(0)-\varepsilon)(\theta+b)\}$, we find

$$
A \leq \int_{B(0, s)}\left(\frac{2^{a}}{a}|x|^{-a}\right)^{1 /(2 a)} d x \leq\left(\frac{2^{a}}{a}\right)^{1 /(2 a)} \int_{\mathbf{B}}|x|^{-1 / 2} d x \leq C a^{-1 /(2 a)},
$$

so that we have by $(\mathrm{P} 2)$

$$
A^{-p(x) / p(0)} \leq C A^{-1+c \varepsilon / p(0)} \quad \text { for } x \in B(0, s) \text { and some constant } c>0
$$

and

$$
A^{\varepsilon / p(0)} \leq C \varepsilon^{-(b+\theta)}
$$


Hence we have

$$
\begin{aligned}
& \int_{B(0, s)}\left(\varepsilon^{\theta / p(0)} f(x)\right)^{p(x)-\varepsilon} d x \\
& \leq C\left\{\varepsilon^{k p(0)} A^{-(p(0)-(1+c) \varepsilon) / p(0)} \int_{B(0, s)}\left(\log \frac{2}{|x|}\right)^{(p(0)-\varepsilon)(\theta+b) / \varepsilon} d x\right. \\
& \left.+\varepsilon^{\theta} A^{\varepsilon / p(0)} \int_{B(0, s)} f(x)^{p(x)}\left(\log \frac{2}{|x|}\right)^{-(\theta+b)} d x\right\} \\
& \leq C\left\{\varepsilon^{k p(0)} A^{\varepsilon(1+c) / p(0)}+\varepsilon^{\theta} A^{\varepsilon / p(0)} \int_{B(0, s)} f(x)^{p(x)}\left(\log \frac{2}{|x|}\right)^{-(\theta+b)} d x\right\} \\
& \leq C\left\{\varepsilon^{k p(0)} A^{\varepsilon(1+c) / p(0)}+\varepsilon^{\theta+b} A^{\varepsilon / p(0)}\right\} \leq C\left\{\varepsilon^{k p(0)-(b+\theta)(1+c)}+1\right\} .
\end{aligned}
$$

If we take $b$ and $k$ such that $k p(0)-(b+\theta)(1+c) \geq 0$, then the present case is obtained.

If $A \leq 1$, then we obtain by (P2)

$$
\begin{aligned}
& \int_{B(0, s)}\left(\varepsilon^{\theta / p(0)} f(x)\right)^{p(x)-\varepsilon} d x \\
& \leq \int_{B(0, s)}\left(\log \frac{2}{|x|}\right)^{(\theta+b)(p(x)-\varepsilon) / \varepsilon} d x+\int_{B(0, s)}\left(\varepsilon^{\theta / p(0)} f(x)\right)^{p(x)-\varepsilon}\left(\frac{\varepsilon^{\theta / p(0)} f(x)}{(\log (2 /|x|))^{(\theta+b) / \varepsilon}}\right)^{\varepsilon} d x \\
& \leq C+\int_{B(0, s)}\left(\varepsilon^{\theta / p(0)} f(x)\right)^{p(x)}\left(\log \frac{2}{|x|}\right)^{-(\theta+b)} d x \\
& \leq C\left\{1+\varepsilon^{\theta} \int_{B(0, s)} f(x)^{p(x)}\left(\log \frac{2}{|x|}\right)^{-(\theta+b)} d x\right\} \leq C\left\{1+\varepsilon^{\theta}\left(\log \frac{2}{s}\right)^{-b}\right\} \leq C,
\end{aligned}
$$

which completes the proof.

Given $f$ on $\mathbf{R}^{n}$, recall the definition of the symmetric decreasing rearrangement of $f$ by

$$
f^{\star}(x)=\int_{0}^{\infty} \chi_{E_{f}(t)^{\star}}(x) d t
$$

where $E^{\star}=\{x:|B(0,|x|)|<|E|\}$ and $E_{f}(t)=\{y:|f(y)|>t\}$; see Burchard [6].

Theorem 10.2. There exists a constant $C>0$ such that

$$
\left\|f^{\star}\right\|_{\overline{\mathcal{H}}_{\{0\}}^{p(\cdot), \infty, \theta}(\mathbf{B})} \leq C\left\|f^{\star}\right\|_{L^{p(\cdot)-0, \theta}(\mathbf{B})}
$$

for all measurable functions $f$ on $\mathbf{B}$.

Proof. Let $f$ be a nonnegative measurable function on $\mathbf{B}$ such that $\left\|f^{\star}\right\|_{L^{p(\cdot)-0, \theta}(\mathbf{B})} \leq$ 1. Note that

$$
\int_{\mathbf{B} \backslash B(0, t / 2)}\left(\varepsilon^{\theta / p(0)} f^{\star}(x)\right)^{p(x)-\varepsilon} d x \leq 1
$$


for all $0<t<1$ and $\varepsilon=\left(p^{-}-1\right)(\log 2) / \log (2 / t)$. We have

$$
\begin{aligned}
& \int_{\mathbf{B} \backslash B(0, t)}\left(\varepsilon^{\theta / p(0)} f^{\star}(x)\right)^{p(x)} d x \\
& \leq C\left(\frac{1}{|B(0, t) \backslash B(0, t / 2)|} \int_{B(0, t) \backslash B(0, t / 2)} f^{\star}(x) d x\right)^{\varepsilon} \int_{\mathbf{B} \backslash B(0, t)} \varepsilon^{\theta p(x) / p(0)} f^{\star}(x)^{p(x)-\varepsilon} d x
\end{aligned}
$$

since $f^{\star}$ is radially decreasing. Set

$$
I=\frac{1}{|B(0, t) \backslash B(0, t / 2)|} \int_{B(0, t) \backslash B(0, t / 2)} f^{\star}(x) d x
$$

and

$$
J=\left(\frac{1}{|B(0, t) \backslash B(0, t / 2)|} \int_{B(0, t) \backslash B(0, t / 2)} f^{\star}(x)^{p(x)-\varepsilon} d x\right)^{1 /(p(0)-\varepsilon)} .
$$

If $J \geq 1$, then we have by $(10.2)$

$$
\begin{aligned}
I & \leq J+C \frac{1}{|B(0, t) \backslash B(0, t / 2)|} \int_{B(0, t) \backslash B(0, t / 2)} f^{\star}(x)\left(\frac{f^{\star}(x)}{J}\right)^{p(x)-\varepsilon-1} d x \\
& \leq J+C J^{-p(0)+\varepsilon+1} \frac{1}{|B(0, t) \backslash B(0, t / 2)|} \int_{B(0, t) \backslash B(0, t / 2)} f^{\star}(x)^{p(x)-\varepsilon} d x \leq C J
\end{aligned}
$$

by (P2) since $J \leq C t^{-n / p(0)}(\log (2 / t))^{\theta / p(0)}$ for all $0<t<1$ and if $J \leq 1$, then

$$
I \leq 1+\frac{1}{|B(0, t) \backslash B(0, t / 2)|} \int_{B(0, t) \backslash B(0, t / 2)} f^{\star}(x)^{p(x)-\varepsilon} d x \leq C .
$$

Hence

$$
I^{\varepsilon} \leq C\left(t^{-n \varepsilon / p(0)}(\log (2 / t))^{\theta \varepsilon / p(0)}+1\right) \leq C
$$

so that

$$
\int_{\mathbf{B} \backslash B(0, t)}\left(\varepsilon^{\theta / p(0)} f^{\star}(x)\right)^{p(x)} d x \leq C \int_{\mathbf{B} \backslash B(0, t / 2)}\left(\varepsilon^{\theta / p(0)} f^{\star}(x)\right)^{p(x)-\varepsilon} d x \leq C,
$$

which completes the proof.

\section{References}

[1] Adams, D. R.: A note on Riesz potentials. - Duke Math. J. 42, 1975, 765-778.

[2] Adams, D. R., and L. I. Hedberg: Function spaces and potential theory. - Springer, 1996.

[3] Adams, D. R., and J. XiAo: Morrey spaces in harmonic analysis. - Ark. Mat. 50:2, 2012, 201-230.

[4] AlmeidA, A., and D. Drinem: Maximal, potential and singular type operators on Herz spaces with variable exponents. - J. Math. Anal. Appl. 394:2, 2012, 781-795.

[5] Almeida, A., J. Hasanov, and S. Samko: Maximal and potential operators in variable exponent Morrey spaces. - Georgian Math. J. 15, 2008, 195-208.

[6] Burchard, A.: Rearrangement inequalities. - Lecture notes, June 2009.

[7] Burenkov, V. I., A. Gogatishvili, V. S. Guliyev, and R. Ch. Mustafayev: Boundedness of the fractional maximal operator in local Morrey-type spaces. - Complex Var. Elliptic Equ. 55:8-10, 2010, 739-758. 
[8] Burenkov, V. I., A. Gogatishvili, V. S. Guliyev, and R. Ch. Mustafayev: Boundedness of the Riesz potential in local Morrey-type spaces. - Potential Anal. 35:1, 2011, 67-87.

[9] Burenkov, V. I., and H. V. Guliyev: Necessary and sufficient conditions for boundedness of the maximal operator in the local Morrey-type spaces. - Studia Math. 163:2, 2004, 157-176.

[10] Burenkov, V. I., H. V. Guliyev, and V.S. Guliyev: Necessary and sufficient conditions for the boundedness of fractional maximal operators in local Morrey-type spaces. - J. Comput. Appl. Math. 208:1, 2007, 280-301.

[11] Capone, C., and A. Fiorenza: On small Lebesgue spaces. - J. Funct. Spaces Appl. 3:1, 2005, 73-89.

[12] Chiarenza, F., and M. Frasca: Morrey spaces and Hardy-Littlewood maximal function. Rend. Mat. 7, 1987, 273-279.

[13] Diening, L.: Maximal functions in generalized $L^{p(\cdot)}$ spaces. - Math. Inequal. Appl. 7:2, 2004, $245-254$.

[14] Diening, L.: Riesz potentials and Sobolev embeddings on generalized Lebesgue and Sobolev spaces $L^{p(\cdot)}$ and $W^{k, p(\cdot)}$. - Math. Nachr. 263:1, 2004, 31-43.

[15] Diening, L., P. Harjulehto, P. Hästö, Y. Mizuta, and T. Shimomura: Maximal functions in variable exponent spaces: limiting cases of the exponent. - Ann. Acad. Sci. Fenn. Math. $34,2009,503-522$.

[16] Diening, L., P. Harjulehto, P. Hästö, and M. RůžıČKa: Lebesgue and Sobolev spaces with variable exponents. - Lecture Notes in Math. 2017, Springer, Heidelberg, 2011.

[17] Di Fratta, G., and A. Fiorenza: A direct approach to the duality of grand and small Lebesgue spaces. - Nonlinear Anal. 70:7, 2009, 2582-2592.

[18] Fiorenza, A., and J. M. Rakotoson: Some estimates in $G \Gamma(p, m, w)$ spaces. - J. Math. Anal. Appl. 340:2, 2008, 793-805.

[19] Gogatishvili, A., and R. Ch. Mustafayev: Dual spaces of local Morrey-type spaces. Czechoslovak Math. J. 61:3, 2011, 609-622.

[20] Gogatishvili, A., and R. Ch. Mustafayev: New pre-dual space of Morrey space. - J. Math. Anal. Appl. 397:2, 2013, 678-692.

[21] Guliyev, V.S., J. J. Hasanov, and S. G. Samko: Boundedness of the maximal, potential and singular operators in the generalized variable exponent Morrey spaces. - Math. Scand. 107:2, 2010, 285-304.

[22] Guliyev, V. S., J. J. Hasanov, and S. G. Samko: Maximal, potential and singular operators in the local "complementary" variable exponent Morrey type spaces. - J. Math. Anal. Appl. 401:1, 2013, 72-84.

[23] Herz, C.: Lipschitz spaces and Bernstein's theorem on absolutely convergent Fourier transforms. - J. Math. Mech. 18, 1968, 283-324.

[24] Izuki, M.: Fractional integrals on Herz-Morrey spaces with variable exponent. - Hiroshima Math. J. 40:3, 2010, 343-355.

[25] Kováčik, O., and J. RÁkosník: On spaces $L^{p(x)}$ and $W^{k, p(x)}$. Czechoslovak Math. J. 41, $1991,592-618$.

[26] Mizuta, Y., E. Nakai, T. Ohno, and T. Shimomura: Riesz potentials and Sobolev embeddings on Morrey spaces of variable exponent. - Complex Var. Elliptic Equ. 56, 2011, 671-695.

[27] Mizuta, Y., and T. Ohno: Duality of Herz-Morrey spaces of variable exponent. - In: The Proceedings on Potential Theory in Sapporo, Japan, 2013 (to appear).

[28] Mizuta, Y., T. Ohno, and T. Shimomura: Sobolev embeddings for Riesz potential spaces of variable exponents near 1 and Sobolev's exponent. - Bull. Sci. Math. 134, 2010, 12-36. 
[29] Mizuta, Y., and T. Shimomura: Sobolev embeddings for Riesz potentials of functions in Morrey spaces of variable exponent. - J. Math. Soc. Japan 60, 2008, 583-602.

[30] Morrey, C. B.: On the solutions of quasi-linear elliptic partial differential equations. - Trans. Amer. Math. Soc. 43, 1938, 126-166.

[31] NAKAI, E.: Hardy-Littlewood maximal operator, singular integral operators and the Riesz potentials on generalized Morrey spaces. - Math. Nachr. 166, 1994, 95-103.

[32] Peetre, J.: On the theory of $L_{p, \lambda}$ spaces. - J. Funct. Anal. 4, 1969, 71-87.

[33] RŮŽIČKA, M.: Electrorheological fluids: Modeling and mathematical theory. - Springer-Verlag, Berlin, 2000.

[34] Stein, E. M.: Singular integrals and differentiability properties of functions. - Princeton Univ. Press, Princeton, 1970.

Received 25 March $2013 \bullet$ Revised received 29 July $2013 \bullet$ Accepted 26 August 2013 